\title{
基于分子钳化合物的手性识别研究进展
}

\author{
刘贵君石 浩* \\ (浙江工业大学药学院 杭州 310014)
}

\begin{abstract}
摘要 手性是自然界中广泛存在的现象, 设计合成新型人工受体模拟生物体内的分子识别已经成为生物有机化学和超 分子化学等领域富于挑战性的课题之一. 分子针类人工受体结构易于修饰, 在手性识别和检测中具有广泛的应用前景, 本文对近年来合成的分子钳人工受体以及应用光谱、色谱和质谱等检测手段对其手性识别性能的研究进行了总结, 并 对分子钳人工受体的发展前景作出了展望.
\end{abstract}

关键词 分子钳人工受体; 手性识别; 光谱; 色谱; 质谱

\section{Progress in Chiral Recognition Based on Molecular Tweezers}

\author{
Liu, Guijun Shi, Hao* \\ (College of Pharmaceutical Science, Zhejiang University of Technology, Hangzhou 310014)
}

\begin{abstract}
Chirality is a common phenomenon in nature. Designing and synthesis of novel artificial receptors to emulate the molecular recognition in living organism has become the challenging forefront of bioorganic chemistry and supramolecular chemistry. Molecular tweezers have been widely applied in chiral molecular recognition and detection for its easily modified structure. Molecular tweezers and their chiral recognition properties detected by spectrometry, chromatography and mass spectrometry are summarized in this paper, and the prospect of molecular tweezers is also discussed.
\end{abstract}

Keywords molecular tweezers artificial receptor; chiral recognition; spectrometry; chromatography; mass spectrometry

手性是自然界中广泛存在的现象, 手性对映异构体 的生物活性往往有所差异甚至完全相反, 如 $\beta$-受体阻断 剂普荎洛尔 (Propranolol)、多巴(Dopar)、沙利度氨 (Thalidomide)等手性药物在人体内的药理活性、代谢过 程及毒性存在显著的差异; 另一方面, 手性对映异构体 又具有极其相似的物理化学性质, 使其识别分离具有一 定难度. 基于手性化合物以上两方面的特点, 利用人工 受体模拟生物体内的分子识别现象已经成为生物有机 化学和超分子化学前沿富于挑战性的课题之一 ${ }^{[1]}$.

自 20 世纪 80 年代中期以来, 用于识别各类阴阳离 子、有机分子以及生物分子的人工受体模型不断被合成 出来, 如冠醚类 ${ }^{[2]}$ 、环糊精类、杯芳烃类 ${ }^{[3]}$ 等. 这些人工 受体模型的开发在医药、化工等领域具有广阔的应用前 景 ${ }^{[4]}$. 人工受体大多能提供一个能与底物相互匹配的环 境 ${ }^{[5]}$, 理想的人工受体模型与生物识别一样, 受体与底 物之间通过氢键、静电作用、芳环 $\pi-\pi$ 堆叠作用、范德 华作用及疏水作用等, 建立精确的互补关系, 从而产生
良好的选择性结合，设计合理的人工受体将具有一定的 手性识别性能.

分子钳 (molecular tweezers) 又被称为分子夹 (molecular clips), 一般由隔离基(spacer)连接二个受体 (receptor)作为 “手臂” 构成, 为具有开放式空腔的非环 化合物. 该空腔能够通过非共价键，如氢键、金属配位、 疏水作用力、范德华作用、静电作用、芳环 $\pi-\pi$ 堆叠作 用等与客体分子结合. 不同于大环化合物与客体分子的 结合，分子钳具有两条 “手臂”，可与客体分子的一端 结合, 具有一定的灵活性. 分子钳人工受体是一类结构 较为简单, 且易于修饰的人工受体模型, 在对映选择性 识别方面有着独特的优越性. 分子钳人工受体可方便地 引入一些具有手性环境及空腔的天然产物进行结构修 饰, 形成分子钳主体结构, 该主体结构具有对一对对映 异构体其中之一更加适合的手性识别环境，从而实现对 手性对映异构体的识别分离. 迄今为止, 已有大量的分 子钳人工受体如以胆甾、杯芳烃、芳杂环等作为隔离基

\footnotetext{
* E-mail: shihao@zjut.edu.cn

Received March 23, 2016; revised June 19, 2016; published online July 8, 2016.

Project supported by the Natural Science Foundation of Zhejiang Province (No. LY13B020013).

浙江省自然科学基金(No. LY13B020013)资助项目.
} 
的分子钳化合物被报道合成, 并用于多种对映异构体的 识别分离. 与此同时, 分子钳人工受体手性识别研究方 法也不断报道出来.

\section{1 分子钳化合物的手性识别研究}

目前用于手性识别研究的方法有很多, 较为常用的 有光谱法、质谱法、色谱法等. 其中光谱法主要包括紫 外-可见光谱(UV-vis Spectroscopy)、核磁共振谱(NMR)、

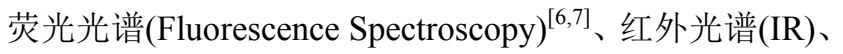
圆二色谱(CD)、X 单晶衍射(X-ray Diffraction)、发射光 谱(Emission Spectroscopy)等; 色谱法主要包括高效液相 色谱(HPLC)、毛细管电泳(CE)、气相色谱 $(\mathrm{GC})$ 、超临界 流体色谱 (SFC) 等; 质谱法主要有电喷雾质谱 (ESI$\mathrm{MS})$ 、化学电离质谱(CI-MS) ${ }^{[8]}$ 、快原子轰击质谱(FAB$\mathrm{MS})^{\text {等 }}{ }^{[9]}$. 以下是近年来一些基于分子钳人工受体的手 性识别研究报道.

\section{1 基于光谱法的手性选择性识别}

当手性分子钳化合物与手性对映异构体分别在溶 液中进行混合时, 由于分子间作用力或空间构型的差异 将引起光谱上的一些细微变化. 通过观测这种细微的变 化辅助以化学计量学分析或与其它光谱联用, 从而对手 性选择剂的识别机理以及作用进行考察. 鉴于光谱法简 单、快捷、准确、灵敏度高, 经常被用于手性识别研 究 ${ }^{[10 \sim 14]}$.

1991 年, Hamilton 等 ${ }^{[15]}$ 设计并合成了以 $R-(-)$-联䒺 骨架作为隔离基的氨基吡啶分子钳 $\mathbf{1}$ 和 $\mathbf{2}$ (图 1), 并通过 ${ }^{1} \mathrm{H}$ NMR 滴定实验考察其对二苯甲酰酒石酸(DBTA)和 二特戊酰基酒石酸(DPTA)的手性选择性. 在晶体中, $D$-(一)-和 $L-(+)$-酒石酸及其衍生物一般二个羧基呈反 式(trans)构型, 而二个羟基呈间扭式(gauche)构型, 如复 合物 3、4 中所示. ${ }^{1} \mathrm{H}$ NMR 滴定结果表明, 人工受体 2 具有比较好的手性选择性, 其在 $\mathrm{CDCl}_{3}$ 中 $D-(-)$-DBTA 的加入使得人工受体 2 酰胺上的 $\mathrm{NH}$ 由于氢键作用向 低场移动了 $\delta 2.8, D$-(一)-DBTA 苯环上 $2,3,4$ 位上的 $\mathrm{H}$ 分别向高场移动了 $\delta 0.32,0.52,0.27$, 这表明 $D-(-)-$ DBTA 的苯环指向荎环, 受到了䒬环的各向异性效应影 响. 此外, 能观察到客体苯甲酰基 2 位的质子和受体 2 萗环 8 位的质子的 NOE 效应, 即形成配合物 $\mathbf{3}$, 而 $L-(+)-D B T A$ 的加入通过 NMR 能观察到氢键作用, 却 没有观察到分子间的 NOE 效应, 即形成配合物 4(图 1). 荧光光谱结果显示, 在 $\mathrm{CH}_{2} \mathrm{Cl}_{2}$ 中受体 2 对 $L-(+)$-DBTA 的结合能力强于 $D-(-)-\mathrm{DBTA}$ (结合常数分别为 $3.6 \times$ $\left.10^{5}, 3.0 \times 10^{5} \mathrm{~L} / \mathrm{mol}\right)$. 而人工受体 2 对 DPTA 的结合能力 恰好相反, 对 $D$ - $(-)$-DPTA 和 $L-(+)$-DPTA 的结合常数 分别为 $1.0 \times 10^{6}$ 和 $3.2 \times 10^{5} \mathrm{~L} / \mathrm{mol}$.
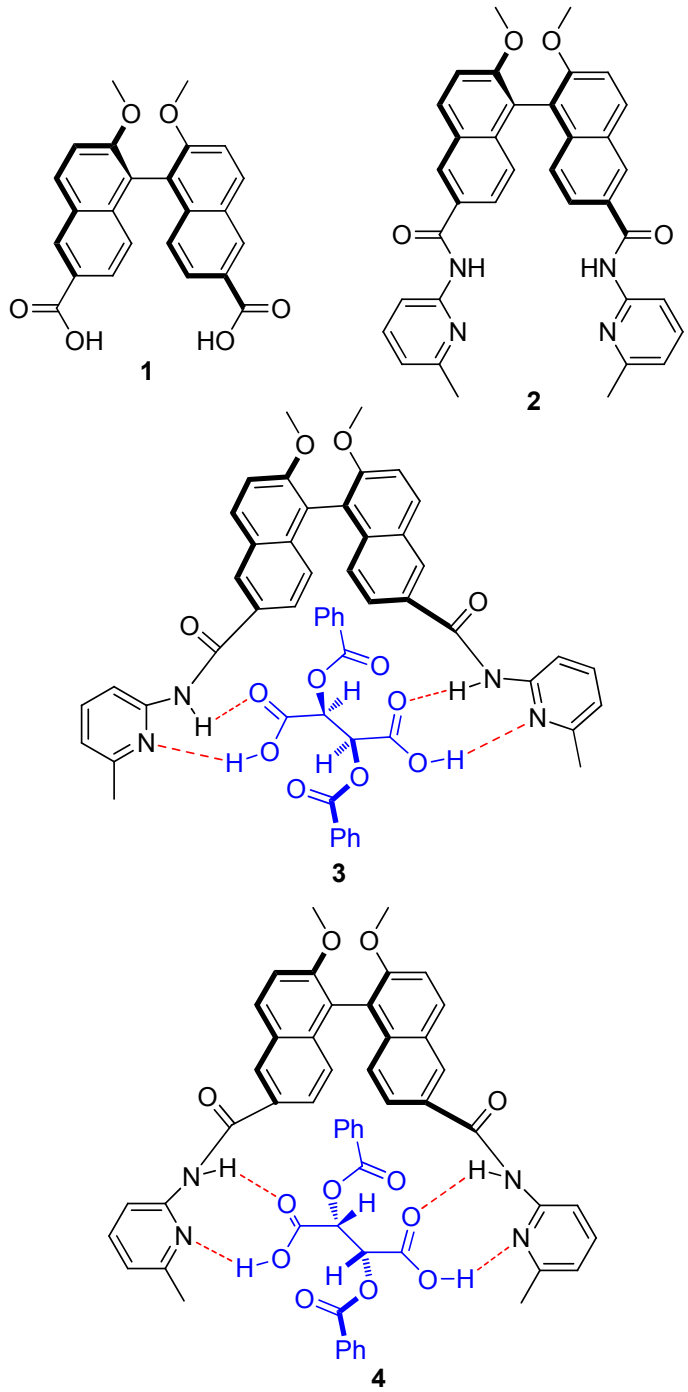

图 1 化合物 1 $\sim 2$ 以及配合物 $3 \sim 4$ 的结构

Figure 1 Structures of compounds $\mathbf{1} \sim \mathbf{2}$ and complexations of $3 \sim 4$

2000 年, Goswami 等 ${ }^{[16]}$ 合成了五个具有一定刚性的 芳杂环分子钳人工受体, 用于识别不同链长的二羧酸, 并认为这类人工受体可作为苂光探针用于检测不同链 长的二羧酸. 通过 ${ }^{1} \mathrm{H}$ NMR 滴定实验考察了其中的外消 旋受体 5 和 6(图 2) 对 $(+)$-樟脑酸的手性识别能力. 实验 表明，外消旋受体 5 和 6 对(+)-樟脑酸(7)有一定的识别 作用, 随着 $(+)$-樟脑酸浓度的增加, 外消旋受体 $\mathbf{5}$ 和 $\mathbf{6}$ 的酰胺氮氢质子原本的一组峰裂分为两组峰，且在不同 的(十)-樟脑酸浓度下, 其化学位移不同, 如图 3 为外消 旋受体 5 在不同的 $(+)$-樟脑酸浓度下的化学位移, 由此 计算得出的结合常数分别为 $2.5 \times 10^{2}$ 和 $3.0 \times 10^{2} \mathrm{~L} / \mathrm{mol}$.

2005 年, Liu 等 ${ }^{[17]}$ 设计并合成了胆甾类分子钳人工 受体 8(图 4), 并通过苂光光谱滴定实验考察了该类人工 受体与二䍭酸阴离子和氨基酸的结合常数, 图 5 为受体 8 和不同浓度 $N$-乙酰- $L$-谷氨酸在甲醇水溶液中的苂光 


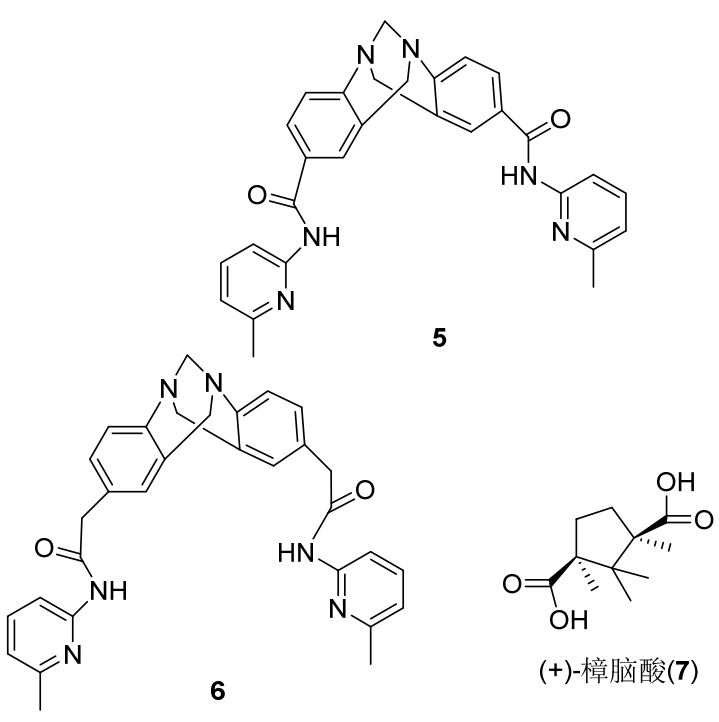

图 2 化合物 $5 \sim 6$ 及 $(+)$-樟脑酸的结构

Figure 2 Structures of compounds $5 \sim \mathbf{6}$ and $(+)$-camphoric acid

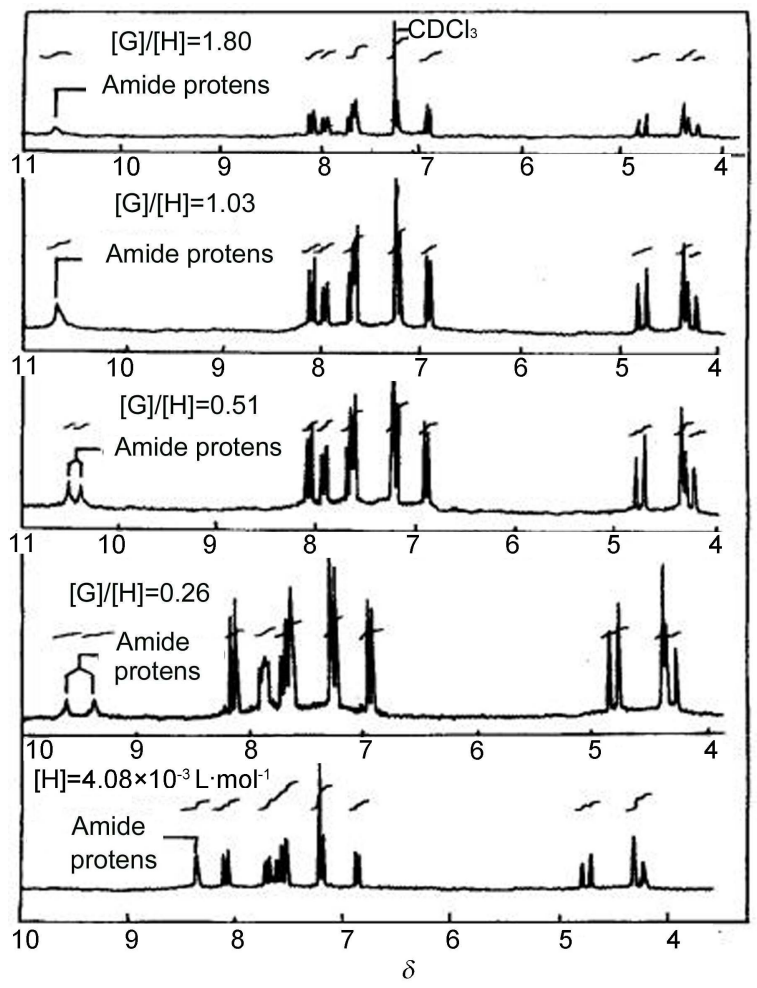

图 3 化合物 5 在不同的 $(+)$-樟脑酸浓度下的化学位移

Figure 3 Chemical shift of compound 5 with different concentrations of $(+)$-camphoric acid solution

光谱图. 实验结果表明, 分子钳 8 对二羧酸阴离子有比 较好的结合能力, 其结合常数均达 $10^{4} \sim 10^{5} \mathrm{~L} / \mathrm{mol}$, 对 $L$-天冬氨酸、 $L$-谷氨酸以及其 $N$-乙酰保护的氨基酸有较 好的识别能力, 尤其是对 $L$-谷氨酸的结合常数达 $5.57 \times$ $10^{6} \mathrm{~L} / \mathrm{mol}$. 分子针 8 对二羧酸阴离子和氨基酸产生识别 作用的位点可能是胆酸 3,7 和 12 位同向的羟基以及“手

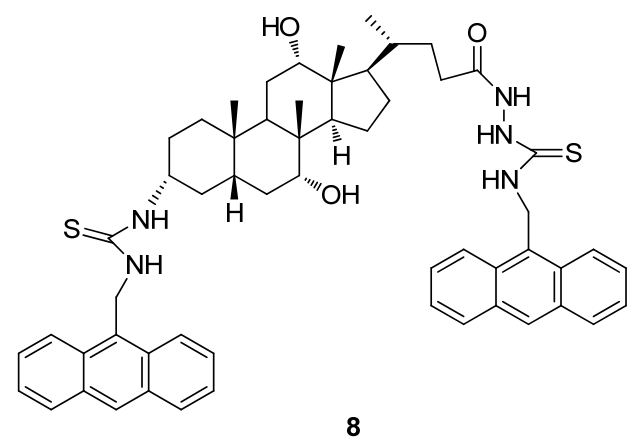

图 4 化合物 8 的化学结构

Figure 4 Structure of compound 8

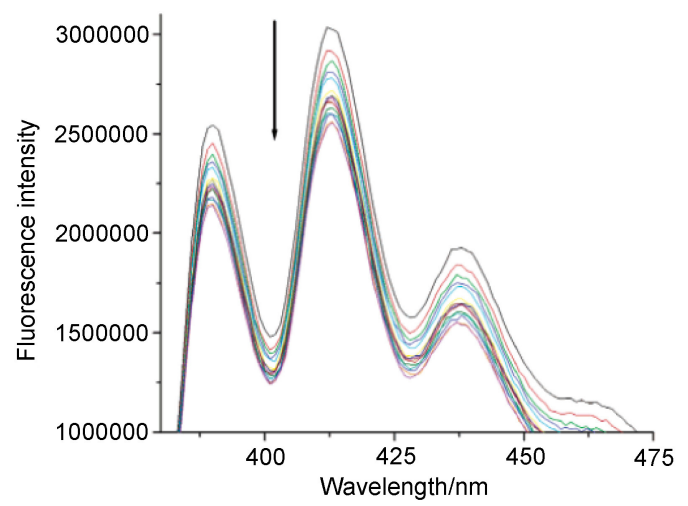

图 5 化合物 $8\left(5.0 \times 10^{6} \mathrm{~mol} / \mathrm{L}\right)$ 和不同浓度 $N$-乙酰- $L$-谷氨酸 在甲醇水溶液中的苂光发射光谱

Figure 5 Emission spectra of $8\left(5.0 \times 10^{6} \mathrm{~mol} / \mathrm{L}\right)$ with different concentrations of $N$-acetyl- $L$-glutamate in aqueous solution $V\left(\mathrm{CH}_{3} \mathrm{OH}\right): V\left(\mathrm{H}_{2} \mathrm{O}\right)=1: 1,0.01 \mathrm{~mol} / \mathrm{L}$ HEPES buffer, $\mathrm{pH}$ 7.4. $\lambda=366$ nm

臂”上的胺基硫嫝基团.

$\mathrm{Li}$ 等 ${ }^{[18]}$ 设计并合成了手性杯[5]芳烃类分子钳 9(图 6), 并通过苂光光谱滴定实验测定该类人工受体对 $\mathrm{Li}^{+}$、 $\mathrm{Na}^{+} 、 \mathrm{~K}^{+} 、 \mathrm{Mg}^{2+} 、 \mathrm{Ba}^{2+} 、 \mathrm{Cu}^{2+}$ 等金属阳离子的结合能力, 结果显示分子钳 9 对 $\mathrm{Cu}^{2+}$ 的选择性远超过其他金属离 子. 同时通过苂光光谱滴定实验考察 $\left[R-(9) \cdot \mathrm{Cu}^{2+}\right]$ 和 $\left[S-(9) \cdot \mathrm{Cu}^{2+}\right]$ 对 $D-(+)$ 木糖、 $D-(-)$ 核糖、 $D-(-)$ 阿拉伯 糖、 $D-(+)$ 葡萄糖以及 $D-(+)$ 葡萄糖-4-内酯等糖类化合 物的手性选择性. 实验结果表明, 在中性水溶液中, 分 子钳 9 仅对 $D-(+)$ 葡萄糖酸内酯具有比较好的手性识别 能力, 其结合常数分别为 $K_{R}=4.45 \times 10^{4} \mathrm{~L} / \mathrm{mol}, K_{S}=$ $1.81 \times 10^{4} \mathrm{~L} / \mathrm{mol}$.

2007 年以来, $\mathrm{He}$ 等 ${ }^{[19-20]}$ 设计并合成了一类双手臂 杯芳烃分子钳化合物 10 14(图 7), 并通过苂光光谱滴 定和 ${ }^{1} \mathrm{H}$ NMR 滴定实验考察其对手性离子以及氨基醇 等客体化合物 $\mathbf{G 1} \sim \mathbf{G 8}$ (图 8)的手性识别能力. 实验结果 表明，这类分子钳化合物对以上客体分子均具有比较好 的手性识别能力, 能与客体分子形成 $1: 1$ 型超分子 


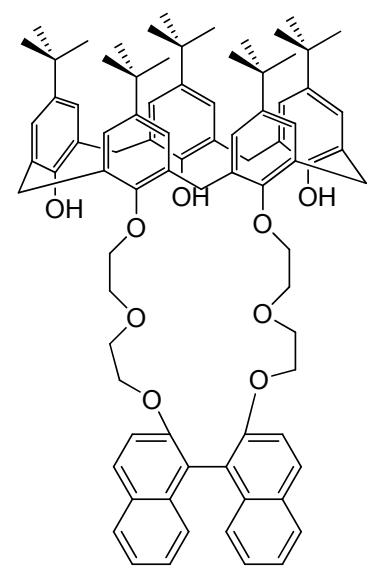

9

图 6 化合物 9 的结构

Figure 6 Structure of compound 9

配合物. 分子钳 10、11 分别对 $N$-叔丁氧羰基保护的丙 氨酸阴离子和扁桃酸有很好的手性选择性, 并认为分子 钳 10、11 具有用作苂光化学传感器的潜力. 杯芳烃的结 构和 $L$-色氨酸的手性中心在手性识别的过程中起着重
要的作用, 其手性识别能力可能主要受主体分子刚性及 多重氢键的共同影响. 如分子钳 11 和 12 与客体的结合 常数高于分子钳 10 , 分子钳 11 和 12 相比分子钳 10 多 两个 $\mathrm{NH}$; 同时, 分子钳 $\mathbf{1 1}$ 与客体的结合常数高于分子 钳 12, 分子钳 11 的刚性比分子钳 12 要好. ${ }^{1} \mathrm{H} N \mathrm{NMR}$ 滴定 结果证实了这一推测, 在客体化合物的存在下, 分子钳 11 的 $\mathrm{NH}$ 化学位移变化比分子钳 10、12 更明显. 苂光 光谱滴定实验发现, 分子钳 13 和 14 与客体分子之间的 作用力主要是主体分子䒺环和客体分子苯环间的 $\pi-\pi$ 堆 叠作用. ${ }^{1} \mathrm{H}$ NMR 滴定结果显示, 在客体化合物氨基醇 的存在下，分子钳 13 和 14 的 $\mathrm{NH}$ 化学位移几乎没有发 生变化, 即多重氢键作用并未在分子钳 13 和 14 与客体 分子产生手性识别作用的过程中起主要作用. 其中分子 钳 14 对苯丙氨醇的手性选择性最好, 其 $K_{L} / K_{D}=4.85$.

2012 年, 冯志强等 ${ }^{[21]}$ 设计并合成了以草酰胺基团 键联双卟啉锌的受体化合物 15(图9), 并通过 $\mathrm{CD}$ 光谱研 究了其对一系列氨基酸酯的手性识别能力. CD 光谱结 果表明, 双卟啉的中心金属离子 $\mathrm{Zn}^{2+}$ 在手性识别过程 中起着不可替代的作用. $L$-型氨基酸酯和双卟啉配位形

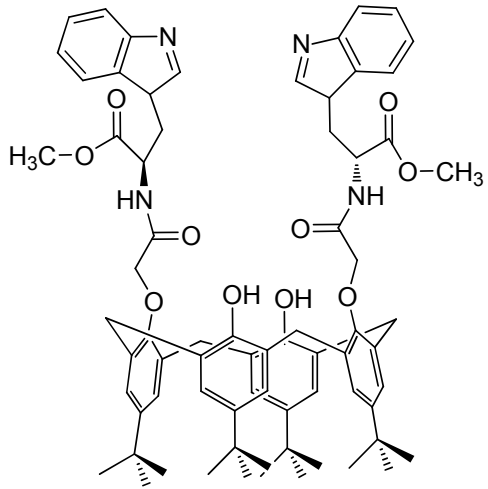

10

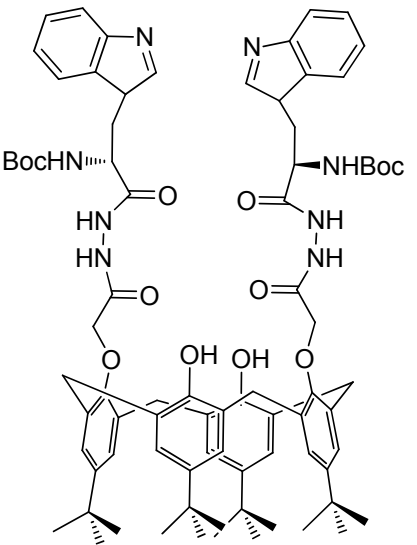

11

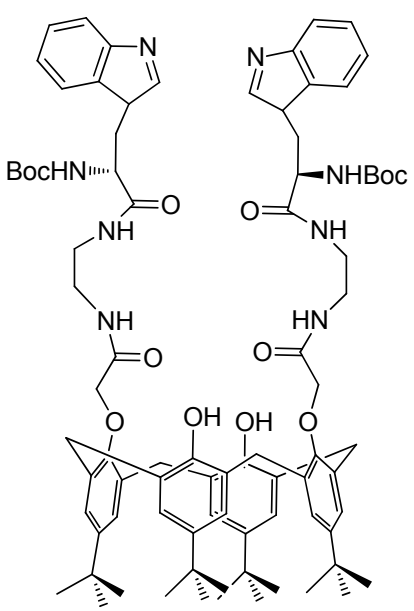

12

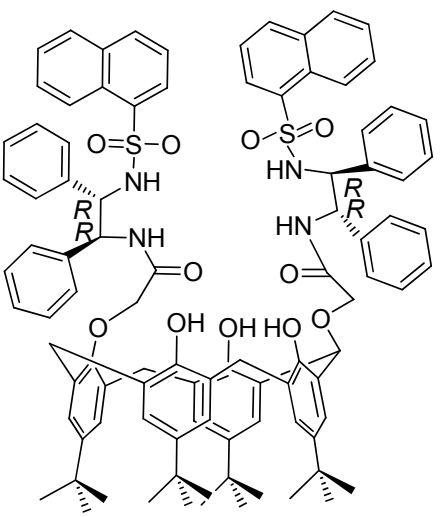

13

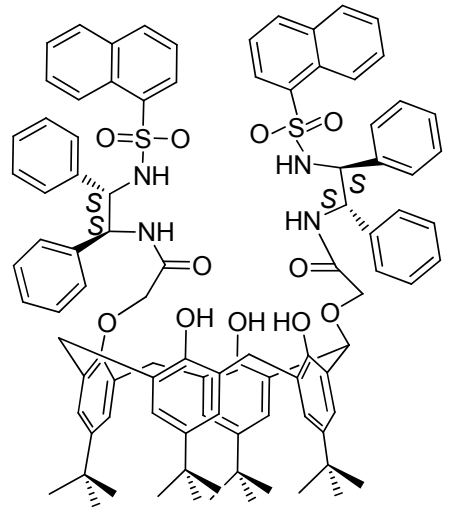

14

图 7 化合物 $10 \sim 14$ 的结构

Figure 7 Structures of compounds $10 \sim 14$ 
<smiles>O=C(O)C(=O)Cl</smiles>

G1<smiles>NC(C(=O)[O-])c1ccccc1</smiles>

G2<smiles>N[C](CO)c1ccccc1</smiles>

G3<smiles>N[C+](CO)Cc1ccccc1</smiles>

G4<smiles>CC(NC(=O)OCc1ccccc1)C(=O)[O-]</smiles>

G5<smiles>O=C([O-])CC(=O)OC(=O)[C@@H](Cc1ccccc1)NC(=O)[O-]</smiles>

G6<smiles>O=C([O-])[C+](O)C(=O)[O-]</smiles>

G8

图 8 客体 $\mathbf{G 1} \sim \mathbf{G 8}$ 的结构

Figure 8 Structures of guests $\mathbf{G 1} \sim \mathbf{G 8}$

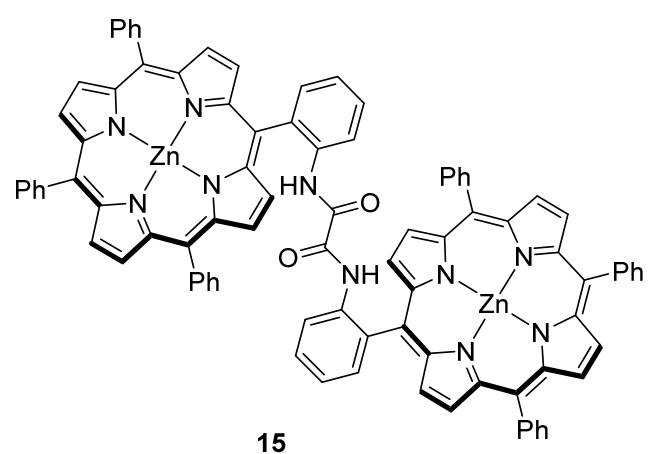

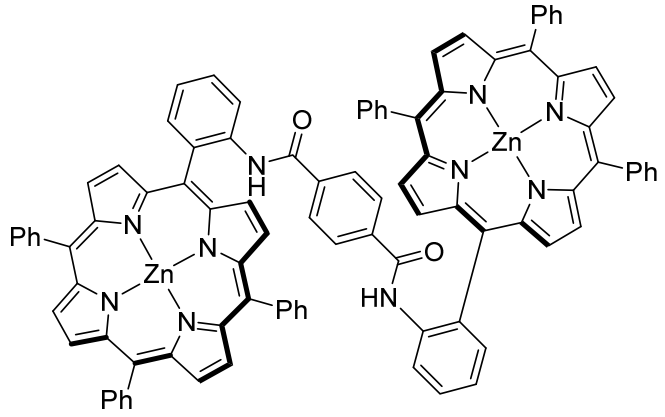

16

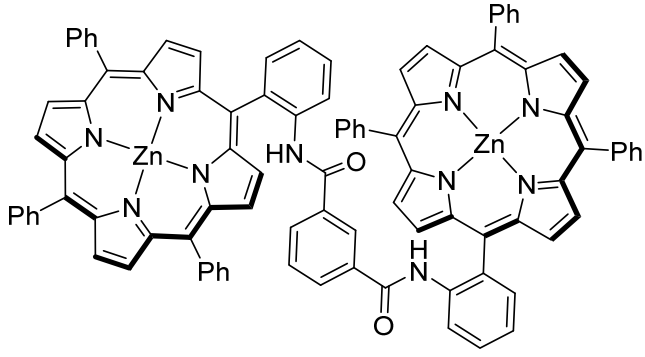

17

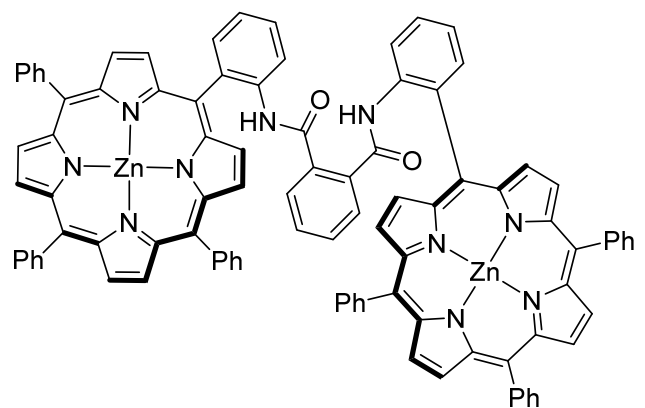

18

图 9 化合物 15 18 的结构

Figure 9 Structures of compounds $15 \sim 18$

成超分子化合物后, 在卟啉的 Soret 带显示正的 Cotton 效应, $D$-型氨基酸酯则在卟啉的 Soret 带显示负的 Cotton 效应. 为探讨双卟啉型受体 15 与氨基酸酯的作用机理, 又通过紫外光谱滴定、 ${ }^{1} \mathrm{H}$ NMR 滴定以及氨基酸酯和卟 啉形成的外消旋手性晶体进行单晶衍射对其识别机理 进行研究, 发现卟啉的中心金属、双卟啉、氨基酸酯的 羰基在手性识别中都起着重要的作用.

2013 年, 蒋佳珣 ${ }^{[22]}$ 设计并合成了一系列刚性酰胺 键联双卟啉锌的钳型分子 $16 \sim 18$ (图 9), 并通过 CD 谱、 $X$ 射线单晶衍射、紫外可见光谱滴定、核磁氢谱以及 DFT/TDDFT 量化计算等方法考察了其对五类氨基酸乙 酯的手性识别能力. 实验结果表明, 钳型分子 $16 \sim 17$ 对 这五类氨基酸乙酯有良好的手性识别能力, 而钳型分子 18 则对这五类氨基酸乙酯均没有手性识别能力. 分子 钳 16 和 17 具有很好的与氨基酸乙酯识别空腔, 而分 子钳 18 由于两个卟啉环之间距离太短而不具备手性识 别氨基酸乙酯的能力. 此外, 双卟啉的金属配位作用、 氨基酸酯上的氨基以及化合物 16 酰胺上羰基所形成的
氢键在手性识别过程中有着重要的作用.

2013 年, Granda 等 ${ }^{[23,24]}$ 设计并合成了含葡萄糖醛酸 单元的分子钳 19(图 10), 并通过 ${ }^{1} \mathrm{H}$ NMR 滴定实验考察 了其在不同的溶剂中对不同的扁桃酸和氨基酸的手性 选择性. 核磁共振谱图表明，分子钳 19 对扁桃酸的 $K_{R} / K_{S}=2.01, S$-扁桃酸与分子钳 19 的两个葡萄糖基产生 作用，而 $R$-扁桃酸仅仅与分子钳 19 的一个葡萄糖基产 生作用, 且分子钳 19 对 $N$-叔丁氧羰基保护的氨基酸阴 离子的 $K_{R} / K_{S}$ 均大于 2.40. 2015 年, Granda 等 ${ }^{[25]}$ 对分子钳 19 的结构进行修饰，引入叔丁基、苯基以及联菱基团, 设计合成了分子钳 $20 \sim 23$ (图 10), 并通过 ${ }^{1} \mathrm{H} N M R$ 滴定 实验考察其对光学纯的苯乳酸阴离子、扁桃酸阴离子、 以及 $N$-叔丁氧羰基保护的色氨酸阴离子的手性选择性, 并与分子钳 19 比较. 实验结果表明, 分子钳 $20 \sim 23$ 的 手性选择性比 19 有所提高. 分子钳 $20 \sim 23$ 的糖基、糖 基上的取代基、客体分子 $\alpha$ 位的氢键供体对手性识别的 结果具有较大影响. 


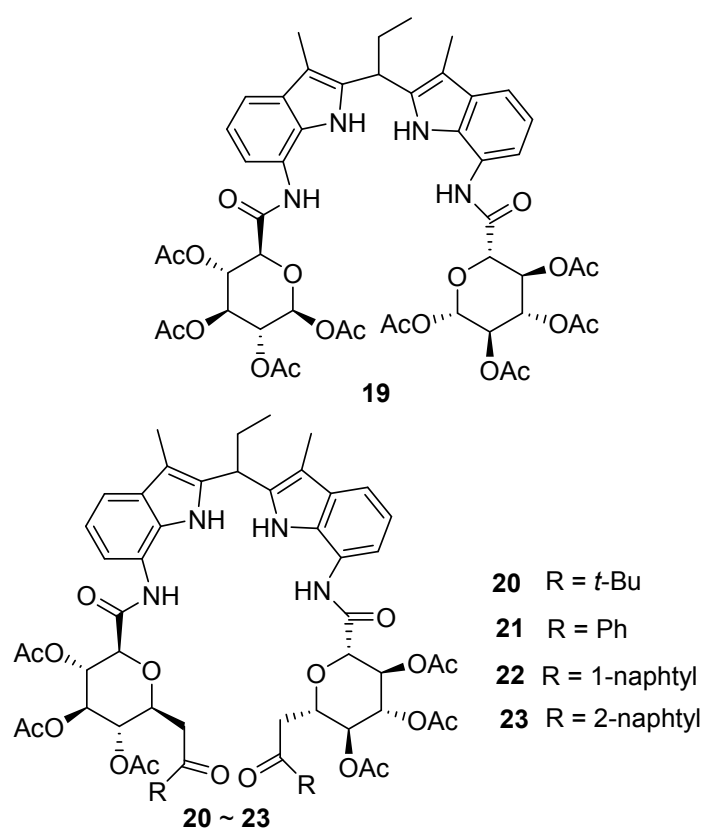

图 10 化合物 19 23 的结构

Figure 10 Structures of compounds 19 23

2015 年, 李正义等 ${ }^{[26]}$ 设计并合成了五种含杯芳烃 和脯氨酸单元的钳型化合物 $24 \sim 28$ (图 11), 并通过 ${ }^{1} \mathrm{H}$ NMR 滴定实验考察其对扁桃酸对映异构体的手性选择 性. ${ }^{1} \mathrm{H}$ NMR 结果显示, 钳型化合物 $\mathbf{2 4} \sim \mathbf{2 8}$ 与扁桃酸客 体分子形成 $1: 2$ 型超分子配合物, 扁桃酸的 $\mathrm{PhCHO}$ 裂 分成为积分面积相等的两个单峰. 以 $24 \sim 26$ 作为主体 时, 发生的裂分分别为 4.2, 28.5, $18.0 \mathrm{~Hz}$. 根据其结合常 数, 计算出分子钳 25 对两种对映体扁桃酸的 $K_{L} / K_{D} \approx$ 94. 其可能的识别机理如图 12, 扁桃酸的 $\mathrm{COOH}$ 先与 25 中的仲胺产生酸碱作用力, 扁桃酸的 $\mathrm{O}=\mathrm{C}$ 与酰胺 $\mathrm{NH}$ 形成分子间氢键, $\mathrm{OH}$ 与 $\mathbf{2 5}$ 中另一分子的脯氨酰胺 的 $\mathrm{O}=\mathrm{C}$ 形成分子间氢键, 从而使两个脯氨酰胺产生相 互协同作用; 扁桃酸苯基相互之间以及它们与杯芳烃空 穴之间存在 $\pi-\pi$ 堆积作用, 进一步增强了主体 25 对扁桃 酸的识别能力.

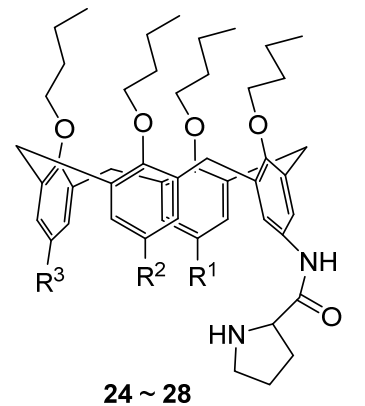

$$
\begin{array}{ll}
24 & R^{1}=R^{2}=R^{3}=H \\
25 & R^{1}=X, R^{2}=R^{3}=H \\
26 & R^{1}=R^{2}=H, R^{3}=X \\
27 & R^{1}=R^{2}==X, R^{3}=H \\
28 & R^{1}=R^{2}=R^{3}=X \\
X=
\end{array}
$$

图 11 化合物 $\mathbf{2 4} \sim \mathbf{2 8}$ 的结构

Figure 11 Structures of compounds $24 \sim 28$

2015 年, Zhang 等 ${ }^{[27]}$ 设计并合成了一系列以环己

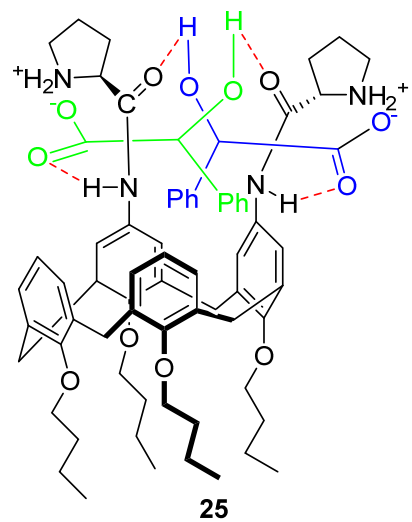

图 12 化合物 $\mathbf{2 5}$ 与扁桃酸可能的识别机理

Figure 12 A possible recognition mechanism for compound 25 and mandelic acid

二胺作为隔离基的分子钳化合物 29 36(图 13)，并通过 ${ }^{1} \mathrm{H}$ NMR 滴定实验考察其对一系列 $\alpha$-羟基酸和 $N$-对甲苯 磺酰基保护的 $\alpha$-氨基酸 $\mathbf{G 9} \sim \mathbf{G 2 0}$ (图 14)的手性识别能 力，以及该分子钳化合物作为手性溶解剂的能力. ${ }^{1} \mathrm{H}$ NMR 滴定实验结果显示, 将化合物与 $\alpha$-羟基酸或 $N$-对 甲苯磺酰基保护的 $\alpha$-氨基酸化合物以 $1: 1$ 混合时，化 合物对 $\alpha$-羟基酸和 $N$-对甲苯磺酰基保护的 $\alpha$-氨基酸均 表现出良好的手性选择性，且随着化合物与 $\alpha$-羟基酸浓 度的增加 $(1: 1$ 混合), $\alpha$-差基酸上 $\alpha-\mathrm{H}$ 的化学位移向高 场移动. 实验还考察了 ${ }^{1} \mathrm{H}$ NMR 滴定的溶剂效应，结果 表明随着 $\mathrm{CDCl}_{3}$ 中大极性的氞代试剂的加入，其手性<smiles>[R]c1cc([R])c(O)c(CNC([R])C(=O)NC2CCCCC2NC(=O)C([R])NCc2cc([R])cc([R])c2O)c1</smiles>

图 13 化合物 29 36 的结构

Figure 13 Structures of compounds 29 $\sim 36$<smiles>[R]c1cc(Br)ccc1C(O)C(=O)O</smiles>

G9 $\mathrm{R}^{1}=\mathrm{H}, \mathrm{R}^{2}=\mathrm{H}$

G10 $\mathrm{R}^{1}=\mathrm{Cl}, \mathrm{R}^{2}=\mathrm{H}$

G11 $R^{1}=H, R^{2}=\mathrm{Cl}$

G12 $R^{1}=H, R^{2}=\mathrm{OCH}_{3}$

$$
\begin{aligned}
& 29 \mathrm{R}=\mathrm{Bn}, \mathrm{R}^{1}=\mathrm{H}, \mathrm{R}^{2}=\mathrm{H} \\
& 30 \mathrm{R}=\mathrm{Bn}, \mathrm{R}^{1}=\mathrm{H}, \mathrm{R}^{2}=\mathrm{Cl} \\
& 31 \mathrm{R}=\mathrm{Bn}, \mathrm{R}^{1}=\mathrm{Br}, \mathrm{R}^{2}=\mathrm{Br} \\
& 32 \mathrm{R}=\mathrm{Bn}, \mathrm{R}^{1}=\mathrm{H}, \mathrm{R}^{2}=\mathrm{OCH}_{3} \\
& 33 \mathrm{R}=\mathrm{Ph}, \mathrm{R}^{1}=\mathrm{H}, \mathrm{R}^{2}=\mathrm{H} \\
& 34 \mathrm{R}=\mathrm{Ph}, \mathrm{R}^{1}=\mathrm{H}, \mathrm{R}^{2}=\mathrm{Cl} \\
& 35 \mathrm{R}=\mathrm{Ph}, \mathrm{R}^{1}=\mathrm{H}, \mathrm{R}^{2}=\mathrm{Br} \\
& 36 \mathrm{R}=\mathrm{Ph}, \mathrm{R}^{1}=\mathrm{H}, \mathrm{R}^{2}=\mathrm{OCH}_{3}
\end{aligned}
$$

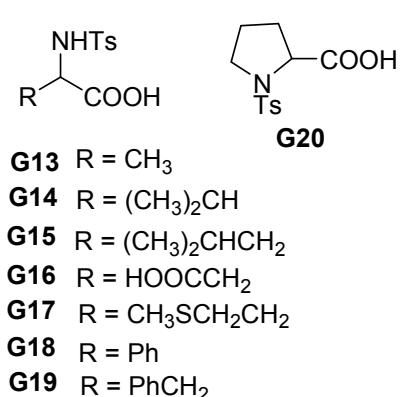

图 14 客体 $\mathbf{G 9} \sim \mathbf{G 2 0}$ 的结构

Figure 14 Structures of guests $\mathbf{G 9} \sim \mathbf{G 2 0}$ 
识别能力逐渐减弱, 且最终消失. 该类分子钳化合物可 作为 $\alpha$-羟基酸的手性溶剂.

González-Mendoza 等 ${ }^{[28]}$ 设计了一类咪唑盐手性离 子液体 37 39(图 15), 且通过 ${ }^{1} \mathrm{H} N \mathrm{NR}$ 滴定实验考察其 对天冬氨酸三乙胺盐和谷氨酸三乙胺盐的手性选择性, 并用 ATR-FTIR 研究其识别机理. 核磁共振结果表明, 受体 37 39 对 $L$-天冬氨酸三乙胺盐的选择性优于 $D$-天 冬氨酸三乙胺盐, 其手性选择性表现为 $38>37 \approx 39$, 受 体 37 39 对谷氨酸三乙胺盐没有表现出手性选择性. 受体 37 在加入 $L$-天冬氨酸三乙胺盐后, 骨架上的 $\mathrm{NH}$ 、 咪唑环 2 位 $\mathrm{H}$ 以及手性碳上的 $\mathrm{H}$ 分别向低场移动了 $\delta$ 1.57、0.74、0.6, 而芳环上的 $\mathrm{H}$ 则向高场移动. 这表明 酰胺、咪唑环 2 位以及隔离基芳环上的氢键作用在手性 识别过程中起重要作用, ATR-FTIR 结果也证实了这一 点.

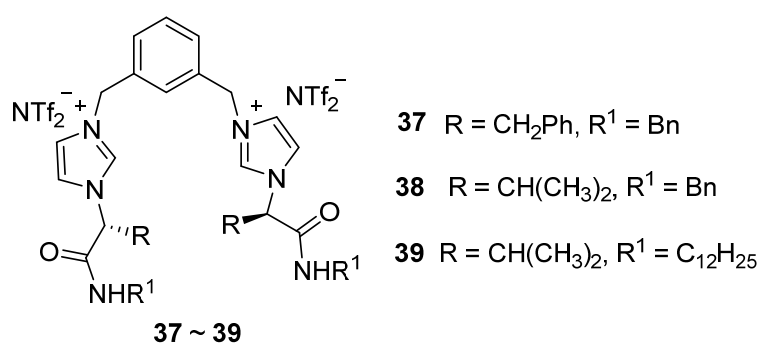

图 15 化合物 37 39 的结构

Figure 15 Structures of compounds $37 \sim 39$

2015 年, Zheng 等 ${ }^{[29]}$ 设计合成了两种四苯乙烯大环 胺类化合物 $(1 S, 2 S)-\mathbf{4 0}$ 和 $(1 R, 2 R)-\mathbf{4 0}$ (图 16), 并通过苂光 光谱和 ${ }^{1} \mathrm{H}$ NMR 滴定考察其对一系列手性酸和 $\alpha$-氨基酸 的手性识别能力. 苂光光谱结果显示, $(1 S, 2 S)$-40 不仅对 手性酸表现出良好的手性识别能力, 如 $(1 S, 2 S)-\mathbf{4 0}$ 对扁 桃酸和樟脑磺酸的手性选择性 $\left(I_{1} / I_{2}\right.$ 分别为 8.4、14.6), 而 且对 $\alpha$-氨基酸表现出良好的手性选择性, 如( $1 S, 2 S)-\mathbf{4 0}$ 对组氨酸的手性选择性 $\left(I_{1} / I_{2}=5.8\right)$. ${ }^{1} \mathrm{H}$ NMR 滴定实验的 结果也证实了这一结论. 当( $1 S, 2 S)-\mathbf{4 0}$ 与 $(R)$-扁桃酸于氯 仿溶液中 $1: 1$ 混合时, $(1 S, 2 S)-40$ 甲基上的氢 $\left(\mathrm{H}_{\mathrm{e}}\right)$ 、苯环 上甲氧基邻位的氢 $\left(\mathrm{H}_{\mathrm{d}}\right)$ 以及扁桃酸手性碳上的氢 $\left(\mathrm{H}_{\mathrm{c}}\right)$ (图 17)分别向低场移动了 $\delta 0.0326 、 0.0477 、 0.0266$, 扁桃酸 苯环邻位上的氢 $\left(\mathrm{H}_{\mathrm{a}}\right)$ 则向高场移动了 $\delta 0.0554,2 \mathrm{D}$ NOESY 谱可观测到 $\mathrm{H}_{\mathrm{e}}$ 与 $\mathrm{H}_{\mathrm{a}}, \mathrm{H}_{\mathrm{e}}$ 与 $\mathrm{H}_{\mathrm{c}}, \mathrm{H}_{\mathrm{d}}$ 与 $\mathrm{H}_{\mathrm{a}}$ 之间存在 NOE 效应; 当(1S,2S)-40 与 $(S)$-扁桃酸 $1: 1$ 混合时, $\mathrm{H}_{\mathrm{a}}$ 向高场移动了 $\delta 0.0451, \mathrm{H}_{\mathrm{d}}$ 向低场移动了 $\delta 0.0441, \mathrm{H}_{\mathrm{e}}$ 与 $\mathrm{H}_{\mathrm{c}}$ 的化学位移几乎没有发生变化, 2D NOESY 谱仅观测 到 $\mathrm{H}_{\mathrm{e}}$ 与 $\mathrm{H}_{\mathrm{c}}$ 之间的 $\mathrm{NOE}$ 效应, 说明 $(R)$-扁桃酸嵌入 $(1 S, 2 S)-\mathbf{4 0}$ 裂穴比 $(S)$-扁桃酸更深. 在手性识别过程中, 酸碱作用力、主客体分子空穴匹配在手性识别过程中有 着重要的作用.

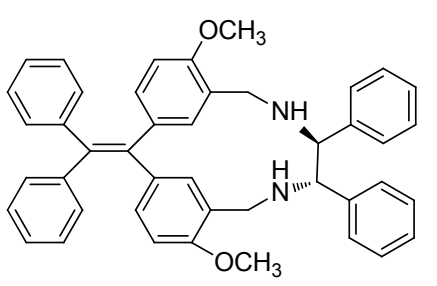

$(1 S, 2 S)-40$

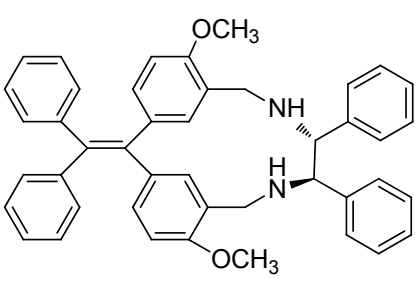

$(1 R, 2 R)-40$

图 16 化合物 $\mathbf{4 0}$ 的结构

Figure 16 Structures of compound $\mathbf{4 0}$

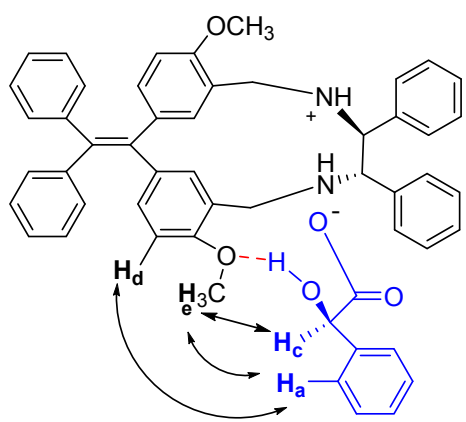

$(1 S, 2 S)-40$ 与 $(R)$-扁桃酸

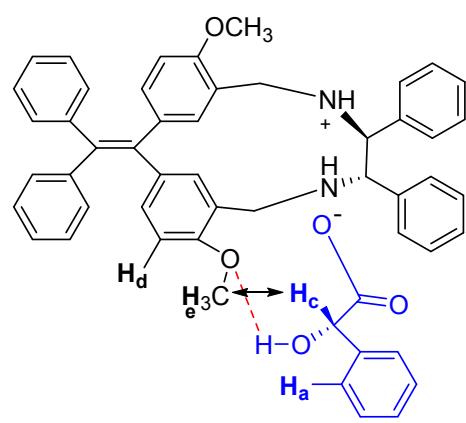

$(1 S, 2 S)-40$ 与 $(S)$-扁桃酸

图 17 化合物 $(1 S, 2 S)-\mathbf{4 0}$ 与扁桃酸的作用

Figure 17 Interactions between compound (1S,2S)-40 and Mandelic acid

Wen 等 ${ }^{[30]}$ 设计并合成了一种用联䒺作为隔离基的 钳型化合物 41(图 18), 并考察其用作苂光探针的能力. 在苂光光谱实验中, 钳型化合物 41 有两个荧光淬灭的 波长, 一个波长对加入的底物浓度很敏感. 另一个波长 对底物的手性很敏感. 钳型化合物 41 在醋酸锌的存在 下，用手性胺，如氨基醇、氨基酸等处理后, 2-蒜胺基团 被取代, 生成的 2-萗胺使得 $\lambda=427 \mathrm{~nm}$ 处的苂光大大增 强, 且该波长下的荧光强度的变化仅与底物浓度有关, 与此同时联菜基团与手性底物结合使得 $\lambda>500 \mathrm{~nm}$ 处的 
荧光大大增强, 且该波长下的苂光强度的变化仅与底物 手性有关. 如图 19 所示: 钳型化合物 $\mathbf{4 1}$ 在醋酸锌的存 在下, 加入 $(1 S, 2 S)$-环已二胺后, 427 和 $528 \mathrm{~nm}$ 处的荧光 强度大大增强，而加入 $(1 R, 2 R)$-环已二胺后，仅 $427 \mathrm{~nm}$ 处的荧光强度大大增强.

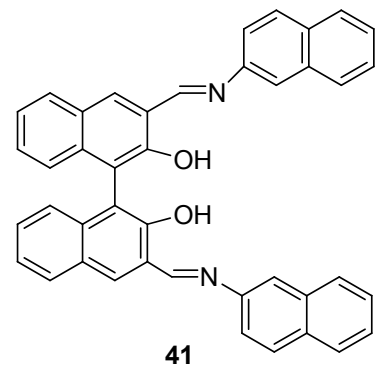

图 18 化合物 41 的结构

Figure 18 Structure of compound 41

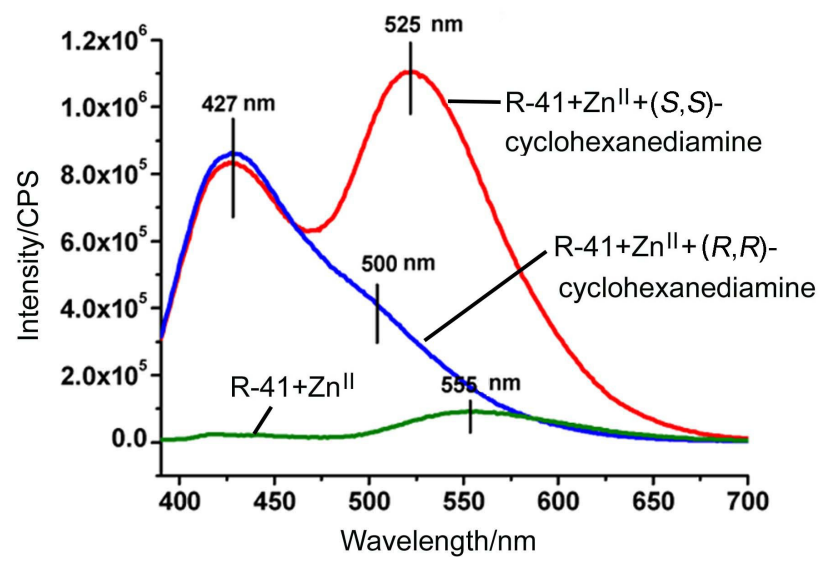

图 19 加入 $\mathrm{Zn}(\mathrm{OAc})_{2}$ 的 41 在环已二胺存在下的荧光光谱 Figure 19 Fluorescence spectra of 41 with $\mathrm{Zn}(\mathrm{OAc})_{2}$ in the presence of cyclohexanediamine

2015 年, Fuentes 等 ${ }^{[31]}$ 设计合成了一种杂环外消旋 化合物 42(图 20), 并通过 ${ }^{1} \mathrm{H} N \mathrm{NMR}$ 滴定实验考察其对氨 基酸衍生物 G21 G34(图 21)的手性选择性. ${ }^{1} \mathrm{H}$ NMR 滴 定结果表明, 分子钳 42 对大部分的氨基酸衍生物具有 良好的手性识别能力, 其中分子钳 $\mathbf{4 2}$ 对 5 -甲基-2-(3-苯 基-腿基)-己酸和 2-(3-环己基硫艮基)-丙酸的 $K_{\text {rel }}$ 分别 为 6.6、6.0. 在三氟乙酰基 $-L$-苯丙氨酸的存在下, 外消 旋化合物 $\mathbf{4 2}$ 的很多 ${ }^{1} \mathrm{H}$ NMR 信号均发生裂分, 即形成 了两种非对映异构体复合物. (一)-42 对三氟乙酰基苯丙 氨酸可能的识别机理如图 22 所示, (一)-42 和三氟乙酰 基苯丙氨酸在识别过程中有四个氢键作用力. 此外, 发 现(一)-42 的叔丁基与三氟乙酰基 $-L$-苯丙氨酸的 $\alpha-\mathrm{H}$ 之 间存在 NOE 效应，而与三氟乙酰基- $D$-苯丙氨酸的 $\alpha-\mathrm{H}$ 却没有 NOE 效应, 三氟乙酰基- $L$-苯丙氨酸与 $(-)-42$ 的 叔丁基之间的空间位阻作用是产生这种差别的原因.

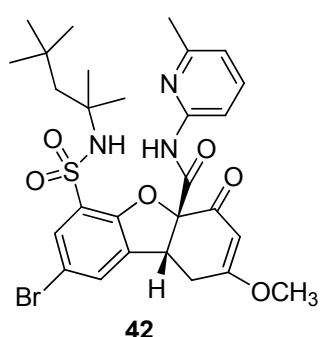

图 20 外消旋化合物 $\mathbf{4 2}$ 的结构

Figure 20 Structure of racemic $\mathbf{4 2}$<smiles>[R]NC([X])NC([R2])C(=O)O</smiles>

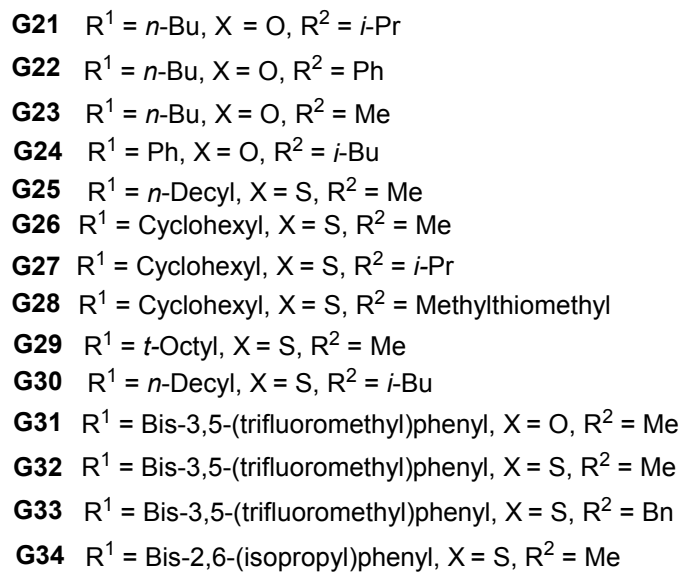

图 21 客体 $\mathbf{G 2 1} \sim \mathbf{G 3 4}$ 的结构

Figure 21 Structures of guests G21 $\sim$ G34

Lhotak 等在 2014 年设计并合成了一种杯 [4]芳烃衍

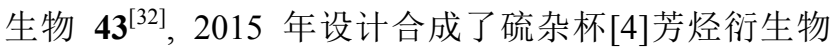
44 ${ }^{[33]}$ (图 23), 并通过 ${ }^{1} \mathrm{H}$ NMR 滴定实验考察其对一系列 氨基酸衍生物、扁桃酸以及氨基醇的手性选择性. ${ }^{1} \mathrm{H}$ NMR 滴定结果显示, 分子钳 $\mathbf{4 3}$ 和 44 对氨基酸衍生物、 扁桃酸以及氨基醇均表现出良好的手性选择性，钳型受 体 43 对 $D$ 型的苯丙氨酸和 $N$-乙酰保护的苯丙氨酸的手 性选择性较好, 其 $K_{D} / K_{L}$ 分别为 $2.86 、 2.16$, 在滴加苯丙 氨酸后, 43 的 $\mathrm{NH}$ 的化学位移发生较大的变化 $(\delta>3.0)$, 而对扁桃酸几乎没有手性识别作用. 钳型受体 44 对 $D$ 型的丝氨醇的手性选择性较好, 其 $K_{D} / K_{L}=3.13$, 对 $(S)$ 扁桃酸的手性选择性较 43 好, 其 $K_{S} / K_{R}=3.13$, 在识别 过程中，适合大小的空穴以及空穴内分子间氢键有着重 要的作用.

\section{2 基于色谱法的手性选择性识别}

色谱法集分离与测定于一体，可实现对映异构体定 性、定量和纯度的测定，是目前比较成熟的手性分析方 法. HPLC 作为一种适用范围比较广泛的手性色谱分离 

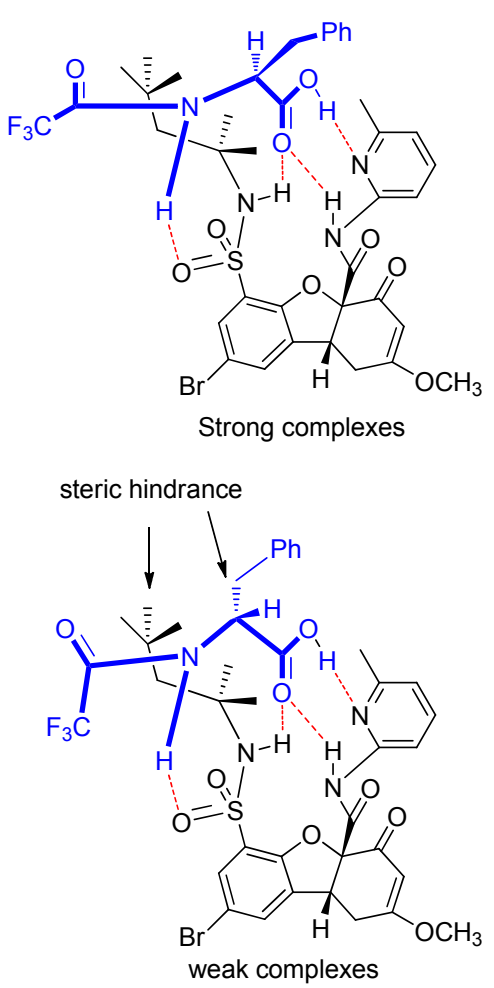

图 $22(-)-42$ 与三氟乙酰基苯丙氨酸可能的识别机理

Figure 22 A possible recognition mechanism for compounds (-)-42 and trifluoroacetylphenylalanine

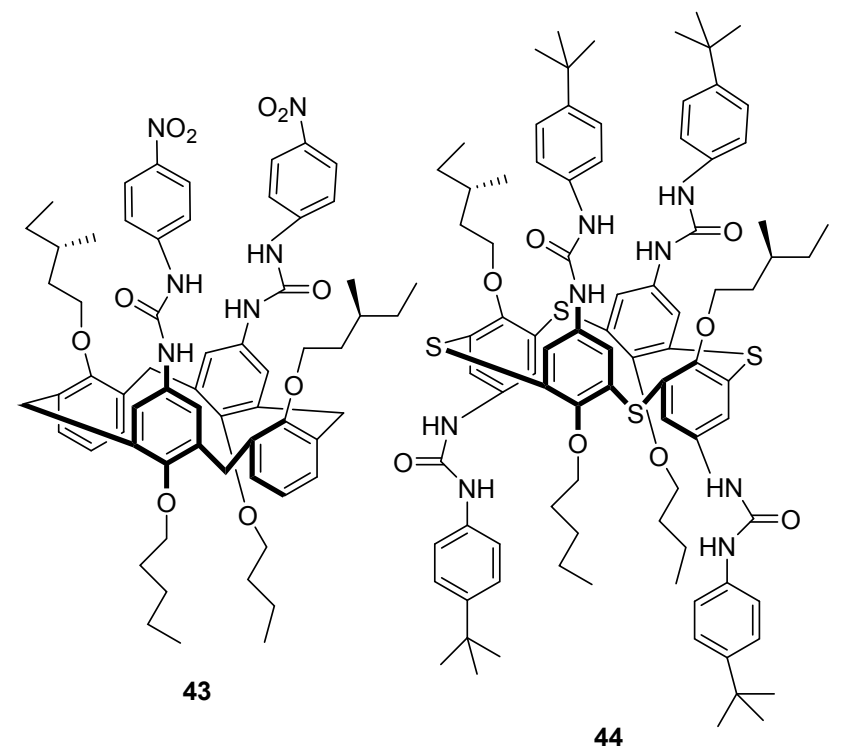

图 23 化合物 $\mathbf{4 3} \sim \mathbf{4 4}$ 的结构

Figure 23 Structures of compounds $43 \sim 44$

方法, 可分为直接法和间接法. 手性色谱分离的直接法 包括手性固定相法(CSP) 和流动相添加法(CMPA), 这两 种方法可以直接而简便地对对映体底物进行拆分和测 定. 手性色谱分离的间接法也称为手性试剂衍生化法 (CDR), 是先将对映异构体用手性试剂进行衍生, 制备 成非对映异构体后再以常规色谱方法进行分离, 然后将 非对映异构体去衍生化得到光学纯的对映体化合物的
方法. 高效毛细管电泳(HPCE)具有分离效率高、分析时 间短、样品和试剂消耗量低的特点，一直广受关注，但 要求其所使用的手性选择剂能与对映体快速络合, 在溶 剂中稳定, 不干扰检测信号. 目前, 毛细管电泳法所用 的手性选择剂主要包括环糊精及其衍生物、手性冠醚、 大环抗生素、手性胶束这四类. 气相色谱法 $(\mathrm{GC})$ 具有速 度快、简单、灵敏的特点, 但要求被分离样品具有挥发 性，由于在气相的高温下，形成的非对映异构体相互作 用能差别变小, 导致手性选择性降低, 所以气相色谱用 于手性识别分离受到一定的限制. 超临界流体色谱法 (SFC)作为液相与气相的补充, 分离对映体常用的手性 固定相有环糊精及其衍生物、手性多糖及其衍生物 等 ${ }^{[34]}$. 但 $\mathrm{SFC}$ 对仪器要求较高, 普及性较差, 这在一定 程度上限制了该技术的应用 ${ }^{[35]}$.

1998 年, Hernfindez 等 ${ }^{[36]}$ 设计合成了一个以螺二芴 作为隔离基, 氨基苯并噁唑作为手性臂的外消旋分子钳 受体 45(图 24), 并通过薄层色谱考察其手性识别能力. TLC 结果表明, 该外消旋受体对酒石酸衍生物具有良好 的手性识别能力. 实验中, 将外消旋体 $\mathbf{4 5}$ 在涂渍了光学 纯的酒石酸衍生物的硅胶板上点样, 并以氯仿/甲醇 $(V: V=98: 2)$ 作为展开剂, 碘显色显示为两个点 $\left(R_{\mathrm{f}}=\right.$ $0.2 、 0.6)$, 证明形成了两种不同的非对映异构体配合物. 产生识别作用可能的机理如图 24 所示, 酒石酸衍生物 可进入 45 的空穴内, 并在空穴中与 45 通过分子间氢键 产生相互作用.
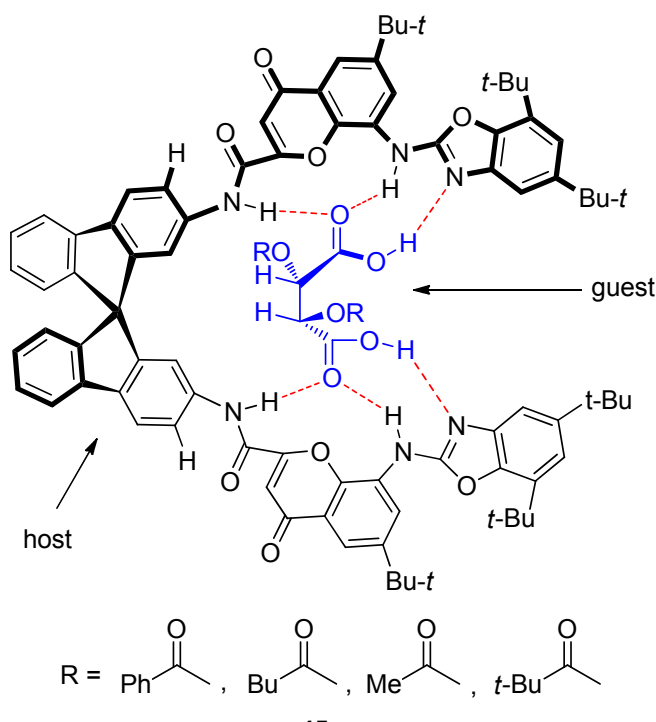

45

图 24 化合物 $\mathbf{4 5}$ 及其与酒石酸衍生物结合的的结构

Figure 24 Structure of compound $\mathbf{4 5}$ and its associate with tartaric acid derivatives

2000 年, Martin 等 ${ }^{[37}$ 设计合成了分子钳外消旋体 46 49(图 25), 并与光学纯的二酮哌嗪在 $\mathrm{CDCl}_{3}$ 中进行 
${ }^{1} \mathrm{H}$ NMR 竞争滴定实验. 结果表明, 分子钳化合物 46 49 对光学纯的二酮哌嗪具较好的有手性识别能力, 其 $K_{\mathrm{rel}}$ 分别为 1.5, 2.2, 2.7 和 6.3. 同时通过薄层色谱(TLC) 实验对这一结果进行验证, 将外消旋体 $46 \sim 49$ 点样在 涂渍了二酮哌嗪的硅胶板上, 以二氯甲烷/乙醚 $(V: V=$ $1: 1)$ 作为展开剂, 外消旋体 46 是一个点, 而外消旋体 $47 \sim 49$ 是两个点, 其比移值分别为 $R_{\mathrm{f}}=0.41 、 0.45, R_{\mathrm{f}}=$ $0.42 、 0.50$ 和 $R_{\mathrm{f}}=0.31 、 0.52$. 外消旋体 47 对二酮哌嗪 可能的识别机理如图 26, 二酮哌嗪与 47 在分子的空穴 内有多重氢键作用, 其分子间氢键作用力随着 47 空间 构型的不同而有强有弱.<smiles>CCOC(=O)C1=CC(NC(=O)c2ccc(CP(=O)(O)OCC)cc2)=C2NC(=O)C(C)(Br)C(=O)c3cccc(c3O2)C1=O</smiles>

46<smiles>CCOC(=O)c1cc(Nc2cccc(Cc3ccc(CP(N)(=O)OCC)cc3)c2)c2oc3c4c(ccc3c(=O)c2c1)C(=O)C(C)(Cc1ccccc1)C(=O)N4</smiles>

47

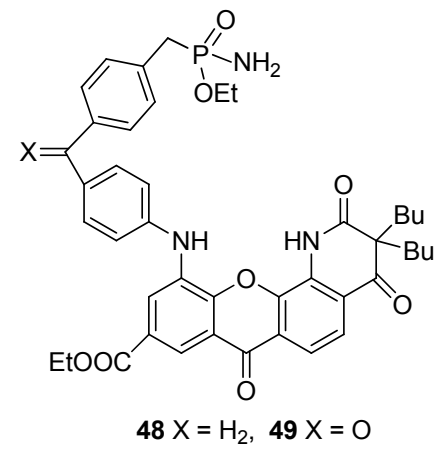

图 25 化合物 $46 \sim 49$ 的结构

Figure 25 Structures of compounds $46 \sim 49$

2005 年, Gazic 等 ${ }^{[38]}$ 设计并合成了七种钳型化合物 50 56 (图 27), 并将其固定在氨丙基硅胶上制备成手性 固定相, 通过 HPLC 验证这七种手性固定相对 13 种 3,4二氢-吡啶酮外消旋体 $\mathbf{G 3 5} \sim \mathbf{G 4 7}$ (图 28)的手性识别能 力. 实验结果表明, 这七种手性固定相均表现出良好的 手性识别能力, 其中以 54 和 56 制备成的手性固定相的 手性识别能力最好. 通过对比以上外消旋体在七种手性 固定相的分离效果以及光学纯的对映异构体在手性固 定相的洗脱顺序, 并结合分子计算机模拟实验发现, 芳 香基团的 $\pi-\pi$ 共轭作用在手性识别中起到的作用较小, 增大手性选择剂上的芳香基团的尺寸来增大分子空腔 的尺寸、增强芳香基的刚性更有利于手性识别能力的提 高, 如以 54、55 和 56 制备成的手性固定相的拆分能力

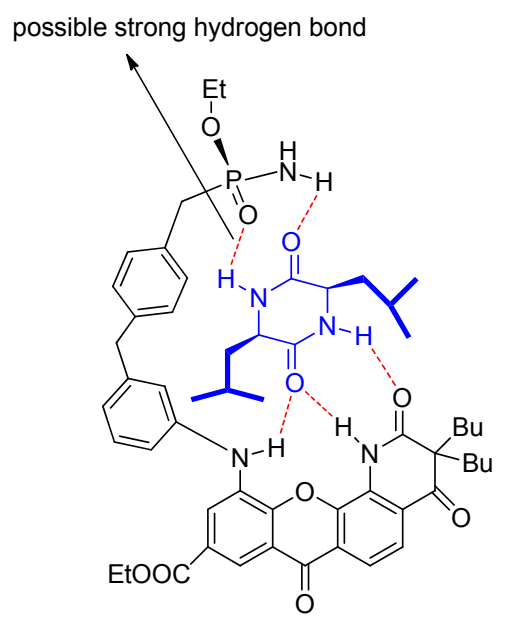

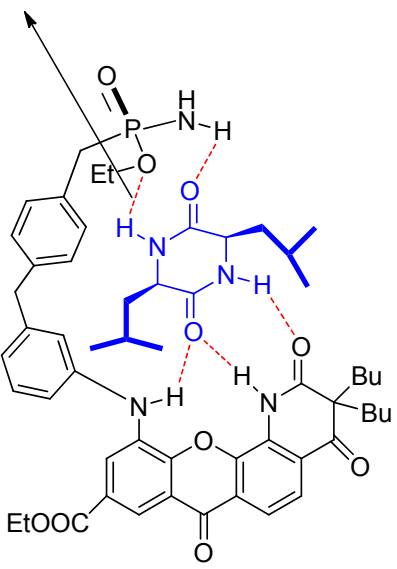

图 26 外消旋 47 与二酮哌嗪可能的识别机理

Figure 26 A possible recognition mechanism for racemic $\mathbf{4 7}$ and diketopiperazine

优于以 52 制备成的手性固定相. 在手性识别过程中，化 合物的空间位阻对其手性识别能力有较大影响，如以 53 制备成的手性固定相的手性拆分能力不如以 54 制备 成的手性固定相, 主要原因是 $\mathbf{5 3}$ 的联荎环 8 位的氢原子 会阻止客体化合物进入手性空穴.
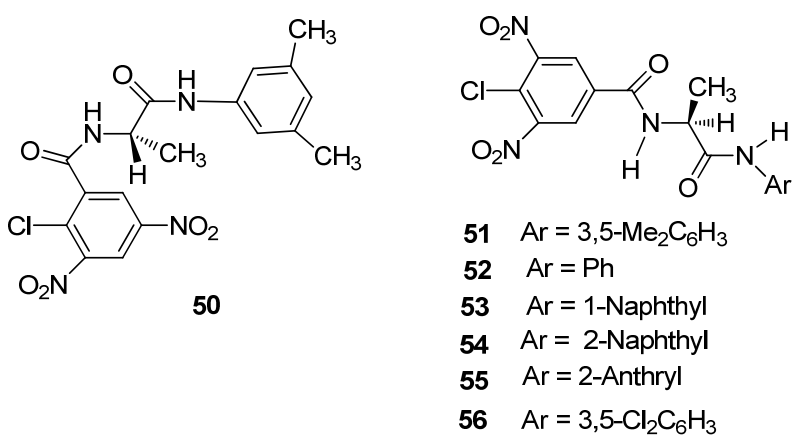

图 27 化合物 50 56 的结构

Figure 27 Structures of compounds $50 \sim 56$ 


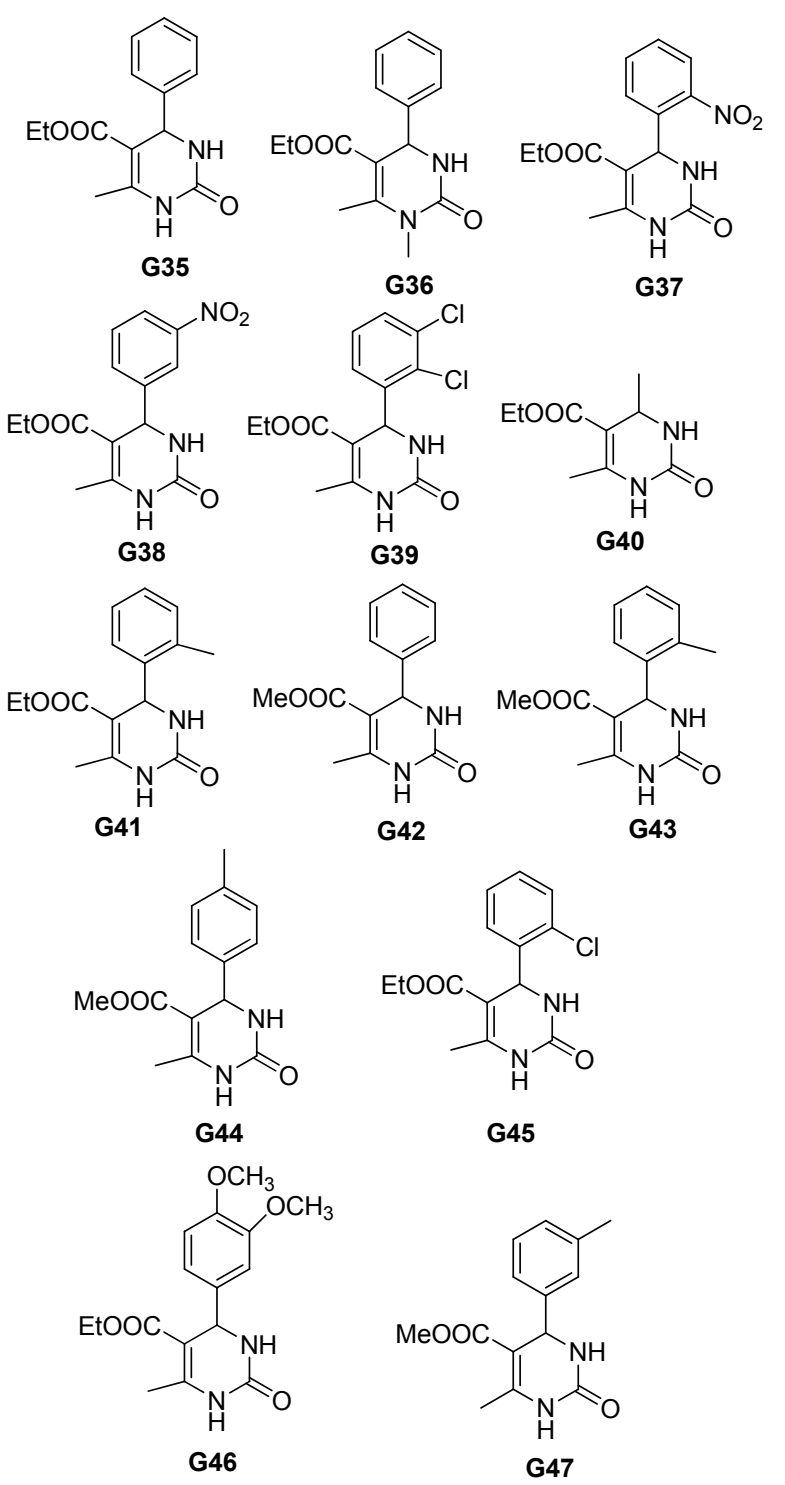

图 28 客体 G35 G47 的结构

Figure 28 Structures of guests $\mathbf{G 3 5} \sim \mathbf{G 4 7}$

2009 年, Choi 等 ${ }^{[39]}$ 将一个含有头孢克洛单元的化合 物 57(图 29)连接在球形硅胶上制备成为手性固定相, 并 通过 HPLC 考察其对外消旋的 N-(3,5-二硝基苯甲酰) $\alpha$ 氨基酸衍生物和外消旋的 3-取代的 1,4-苯并二氮杂-2酮的手性识别能力. 实验结果表明, $S$ 型的 $N-(3,5-$ 二硝 基苯甲酰)- $\alpha$-氨基酸衍生物在该手性柱上被先洗脱下来, 而对于 3-取代的 1,4-苯并二氮杂-2-酮而言, 则是 $R$ 型的 对映异构体先被洗脱下来. 由化合物 $\mathbf{5 7}$ 制备成的手性 固定相上的 3,5-二甲氧基苯基和外消旋对映异构体上的 苯环之间的 $\pi-\pi$ 供体-受体作用对手性识别具有重要的 作用. 比如分别对外消旋 $N$-(取代苯甲酰)亮氨酸乙酯 G48 G52(图 30)进行拆分, 发现由化合物 57 制备成的 手性固定相对 $\mathbf{G 4 8} \sim \mathbf{G 5 0}$ 有良好的拆分能力, 但无法拆 分 G51 $\sim \mathbf{G 5 2}$, 表明在手性识别过程中 $\mathbf{5 7}$ 上的 3,5-二甲
氧基苯基与客体的苯甲酰基之间的 $\pi-\pi$ 供体-受体作用 有着重要影响. 化合物 $\mathbf{5 7}$ 与 $\mathbf{G 4 8}$ 可能的识别机理如图 31 所示, 主要包括 $\pi-\pi$ 供体-受体作用和氢键作用.<smiles>[2H][C@](NC(=O)c1cc(OC)cc(OC)c1)(C(=O)N[C@H]1C(=O)c2c1ccc(Cl)c2C(=O)O)c1ccccc1</smiles>

图 29 化合物 $\mathbf{5 7}$ 的结构

Figure 29 Structure of compound $\mathbf{5 7}$

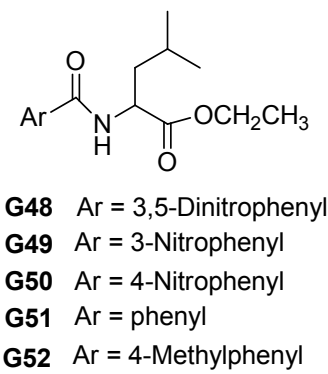

图 30 客体 $\mathbf{G 4 8} \sim \mathbf{G 5 2}$ 的结构

Figure 30 Structures of guests $\mathbf{G 4 8} \sim \mathbf{G 5 2}$

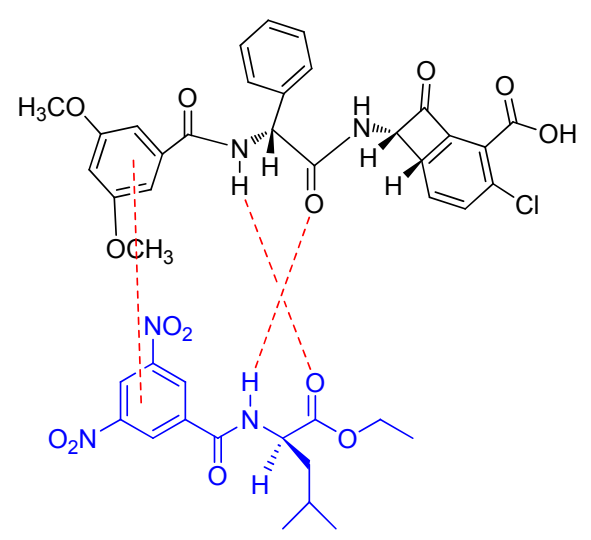

图 3157 与 $\mathbf{G 4 8}$ 可能的识别机理

Figure 31 A possible recognition mechanism for $\mathbf{5 7}$ and $\mathbf{G 4 8}$

2012 年, $\mathrm{Ke}$ 等 ${ }^{[40]}$ 合成了两个含联荎基团和 1,2,3-咪 唑环的化合物 58 和 59(图 32), 并将其制备成手性固定 相. 通过 HPLC 验证这两种手性固定相对一系列的联荎 酚衍生物、吲哚-2-酮衍生物的手性拆分能力, 并通过研 究两种光学纯的对映异构体的洗脱顺序考察其手性识 别的机理. 实验结果显示, 这两种手性固定相对联荎酚 醚类衍生物及 3-苯基吲哚-2-酮类似物表现出良好的拆 分效果, 以 59 制备的手性固定相的手性拆分能力优于 以 58 制备的手性固定相. 分子间氢键、 $\pi-\pi$ 作用以及偶 
极-偶极作用力在手性识别过程中具有重要的作用. 比 如, 含羟基的联萗酚衍生物在两种手性固定相上, $S$ 型 先被洗脱下来, 当羟基被甲氧基取代后洗脱顺序刚好相 反, 这说明在识别过程中分子间氢键具有重要作用, 而 是当羟基被甲氧基取代后, $\pi-\pi$ 作用在识别过程中占据 了主导地位.

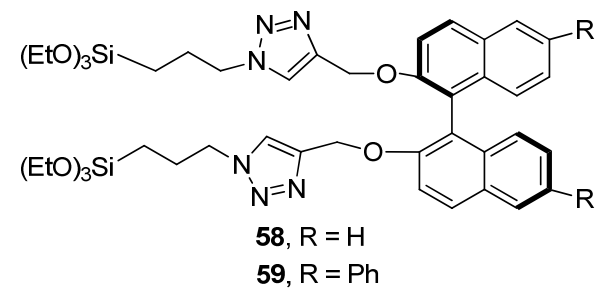

图 32 化合物 $\mathbf{5 8} \sim \mathbf{5 9}$ 的结构

Figure 32 Structures of compounds $\mathbf{5 8} \sim \mathbf{5 9}$

2014 年, $\mathrm{Wu}$ 等 ${ }^{[41]}$ 合成了一种乙烯基咪唑修饰的 $\beta$ 环糊精衍生物 60(图 33), 首次通过微波辐射将环糊精衍 生物 60 固定在 3-(甲基丙烯酰氧)丙基三甲氧基硅烷-纳 米粒子(MPS-MNPs) 表面上, 形成了磁性微球. 同时, 通 过毛细管电泳考察这种磁性微球的手性识别能力. 实验 结果表明, 该磁性微球对丹磺酰氨基酸表现出较好的手 性识别能力, 其对 $L$-丹磺酰氨基酸的保留能力强于 $D-$ 丹磺酰氨基酸. 在手性识别的过程中, 疏水作用、氢键 作用、 $\pi-\pi$ 堆积作用、静电作用等分子间作用力发挥着 重要的作用.

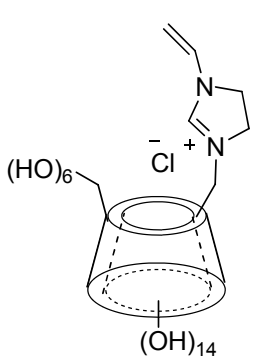

60

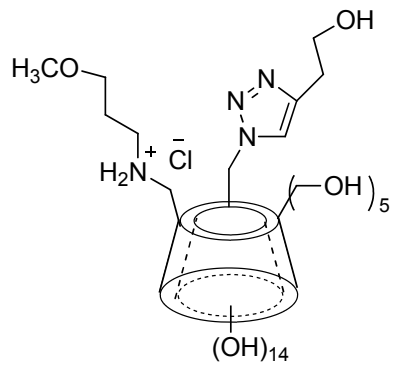

61
图 33 化合物 $60 \sim 61$ 的结构

Figure 33 Structures of compounds $60 \sim 61$

2015 年, Tang 等 ${ }^{[2]}$ 通过点击化学合成了一种以环糊 精作为隔离基的钳型受体 61(图 33), 并通过毛细管电泳 考察其对 7 种两性外消旋体和 13 种酸性外消旋体的手 性选择性. 实验结果显示, 当缓冲溶液 $\mathrm{pH}$ 为 $5.5 \sim 8.0$, 手性选择剂浓度为 $0.5 \sim 7.5 \mathrm{mmol} / \mathrm{L}$ 时, 钳型受体 61 对 除丹磺酰基亮氨酸和丹磺酰基丝氨酸以外的外消旋体 均表现出良好的手性识别能力 $(\alpha \geqslant 1.05)$. 当缓冲溶液 $\mathrm{pH}$ 为 6.0 , 手性选择剂浓度为 $7.5 \mathrm{mmol} / \mathrm{L}$ 时, 钳型受体 61 对邻氯扁桃酸和对羟基扁桃酸的手性拆分能力最高 ( $\alpha$ 分别为 $15.6 、 9.7)$. 同时, 发现三唑基团有利于形成分
子间作用力不同的主客体复合物，从而实现拆分. 此外, 通过 61 与 3-苯基乳酸的 ${ }^{1} \mathrm{H}$ NMR 滴定实验考察其手性 识别的机理, 苯乳酸手性碳上的 $\alpha-\mathrm{H}$ 和三唑环上的 $\mathrm{CH}$ 均向高场移动, $R$ 型和 $S$ 型的 3-苯基乳酸上的 $\alpha-\mathrm{H}$ 的化 学位移从 $\delta 4.56$ 分别变为 $\delta 4.44 、 4.22, R$ 型和 $S$ 型的 3苯基乳酸使三唑环上的 $\mathrm{CH}$ 的化学位移从 $\delta 7.88$ 分别变 为 $\delta 7.77 、 7.80$, 这表明静电作用力在手性识别过程中发 挥着重要的作用.

手性固定相在拆分氨基酸的过程中有着不同的识 别机理, 如通过奎宁的离子交换作用 ${ }^{[43]}$ 和冠醚的络合 作用 ${ }^{[44,45]}$ 对氨基酸进行手性拆分. 2015 年, Wang 等 ${ }^{[46]}$ 设 计合成了两种同时含奎宁和冠醚基团的化合物 62 和 63 (图 34), 再将其连接在 3-巯基丙基硅胶上制备成手性固 定相, 并通过 HPLC 考察了其对一系列手性酸、手性胺 以及氨基酸的识别分离能力, 并与奎宁手性固定相进行 比较. HPLC 结果显示, 对绝大部分手性酸、手性胺以及 氨基酸而言, 以 62 和 63 制备的手性固定相的手性拆分 能力比奎宁手性固定相更为优越. 其中以 62 制备手性 固定相的手性拆分能力优于以 63 制备的手性固定相, 可能是 62 上冠醚基团与奎宁基团之间的距离更有利于 客体的识别. 以 62 制备成的手性固定相与氨基酸的作 用模式如图 35 所示, 氨基酸的氨基与冠醚基团发生络 合作用，羧基与奎宁环的 $\mathrm{N}$ 发生离子交换.

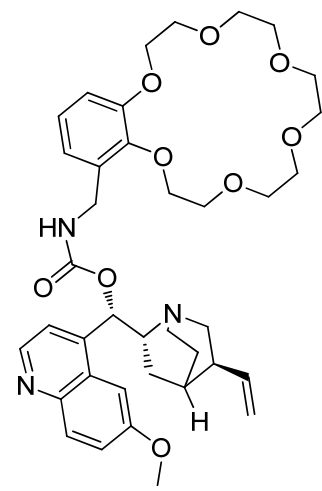

62

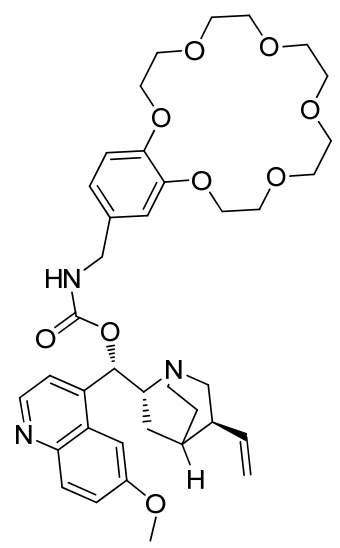

63
图 34 化合物 $62 \sim 63$ 的结构

Figure 34 Structures of compounds $62 \sim 63$

\section{3 基于质谱法的手性选择性识别}

随着质谱技术的不断发展，质谱被引入手性分析 中, 其中包括早期的化学电离质谱(CI-MS)、快原子轰击 质谱(FAB-MS) 以及之后的电喷雾质谱(ESI-MS $)^{[47,48]}$. 化学电离质谱是最早用于手性识别的质谱技术，曾被用 于苦杏仁酸、 $\alpha$-苯乙胺、麻黄碱等手性物质的绝对构型 的确认 ${ }^{[49]}$. 但由于使用化学电离质谱测定样品时, 引入 的试剂气使得样品分子与试剂离子间的作用力变小, 分 


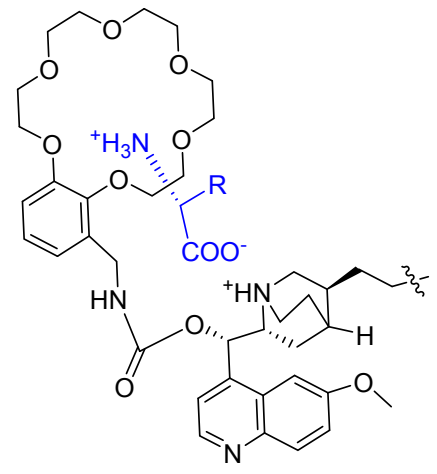

图 35 以 62 制备成的手性固定相与氨基酸的作用模式 Figure 35 Action model between CSP prepared from 62 and amino acid

子离子信号变得很弱, 同时也不适合于对极性强的热敏 性物质进行分析, 使得化学电离质谱的使用受到一定的 限制.

快原子轰击质谱法是随着快原子轰击软电离技术 发展起来的一种质谱方法, 其基本原理是将样品分散于 基质并制成溶液, 涂布了溶液的金属靶被惰性气体中性 原子束轰击, 在轰击中产生的样品离子进入气相, 然后 在电场作用下经过检测器. 快原子轰击质谱法适合用于 对极性大、热稳定性差和不易汽化的样品进行分析. 目 前快原子轰击质谱法在手性识别体系中研究得最多的 是主一客体复合物体系 ${ }^{[50]}$, 其具体的研究方法又分为相 对峰强度法和对映体同位素标记法.

随着主客体理论以及电喷雾(ESI)等软电离质谱技 术的不断发展, 打破了传统质谱电离的方式, 近十几年 以来, 不断有人将电喷雾质谱法(ESI-MS) 用于手性识 别. 电喷雾质谱法具有适用范围广、灵敏度高以及分析 速度快的特点, 成为手性识别的一种重要方法. 电喷雾 质谱法中样品以带电小液滴形式进入电场, 随着溶剂的 不断挥发, 最终样品以单电荷或多电荷的离子形式进入 质量分析器. 电喷雾质谱法中, 单一构型的手性主体分 子与两种构型的客体分子通过一些分子间作用力形成 的非对映体配合物, 由于主客体结合的牢固程度不同, 在被给予相同的解离能时, 能裂解成的碎片离子不同, 所以能在质谱上观察到不同的离子丰度和裂解行为. 根 据其识别机制的不同, 又将电喷雾质谱法的识别方法主 要分为对映体标记法、离子-分子反应法、动力学方法 和其他方法.

1995 年, Habata 等 ${ }^{[51]}$ 设计并合成了十三种含芳环的 手性分子钳化合物, 并通过 FAB-MS 对映体同位素标记 法考察了其对十三种氨基酸酯的手性识别能力. 实验结 果表明, 大部分主体化合物对氨基酸酯表现出一定的手 性选择性 $\left(I_{R} / I_{S-d n}=0.5 \sim 5.4\right)$. 令人遗憾的是, 其中的化 合物 64 和 65(图 36)对氨基酸酯类化合物几乎没有手性
选择性. 然而其中的主体 66 却对大多数 $R$ 型氨基酸酯 表现出优越的手性选择性 $\left(I_{R} / I_{S}=3.2 \sim 5.4\right)$, 如图 37 是 蛋氨酸甲酯与 66 形成的配合物的 FAB-MS 图, 主体 66 与 $R$ 型的蛋氨酸甲酯配合物 $\left[\left(\mathrm{H}+\mathrm{G}_{\mathrm{R}}\right)^{+}\right]$的离子峰度高于 主体 66 与 $S$ 型的蛋氨酸甲酷配合物 $\left[\left(\mathrm{H}+\mathrm{G}_{\mathrm{S}}\right)^{+}\right]$. 此外, 还通过重复试验及主客体复合物在溶液中的热力学稳 定性实验, 再次验证了将 FAB-MS 用作手性识别的优越 性和可靠性.
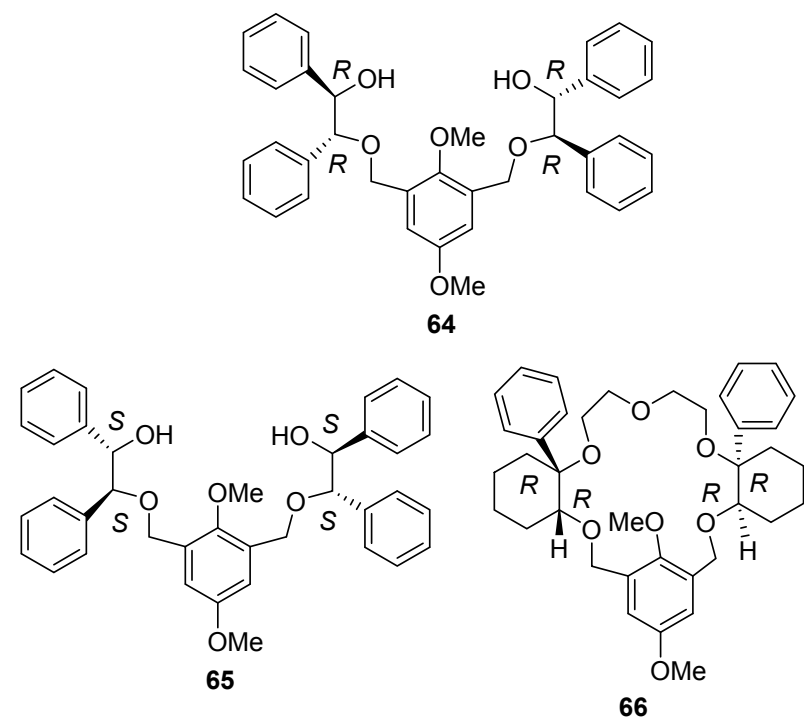

图 36 化合物 64 66 的结构

Figure 36 Structures of compounds $64 \sim 66$

2005 年, Liu 等 ${ }^{[52]}$ 设计并合成了一系列手性三聚氧 胺衍生物 67 75(图 38), 首次通过 ESI-MS 考察其对十 九种天然的蛋白质氨基酸的手性识别能力, 并对其手性 识别机理进行研究. 质谱结果表明, 这类手性三聚氧胺 衍生物对这十九种氨基酸表现出优越的手性识别能力, 如图 39 为苯丙氨酸与 $67 \mathrm{a}$ 形成两种配合物的 ESI 谱. 含 有不同取代基和不同手性中心的化合物对不同的氨基 酸表现出不同的识别能力, 比如 $R$ 构型的三聚氧胺衍生 物 67a 对苯丙氨酸的选择性恰好与 $67 \mathrm{~b}$ 对苯丙氨酸的选 择性相反. 实验结果表明，电荷密度、氢键作用、 $\pi-\pi$ 共 轭作用力以及三聚氰胺衍生物与氨基酸的空间相互作 用在手性识别中表现出重要的作用.

2009 年, Shizuma 等 ${ }^{[33]}$ 设计并合成了一系列共二十 一个分子钳型混合物 76(图 40), 并在不将混合物进行分 离的情况下，通过 FAB-MS 考察了这 21 种结构的混合 物对八种氨基甲酸异丙酯盐酸盐的手性识别能力. 实验 发现部分主体化合物对氨基甲酸异丙酯盐酸盐表现出 良好的手性选择性，表现为 $I_{R} / I_{S-\mathrm{dn}}>1.4$ 或 $I_{R} / I_{S-\mathrm{dn}}<0.71$. 其中含有取代基 $R^{3} 、 R^{5}$ 和 $R^{6}$ 的分子钳化合物手性选择 性较好. 

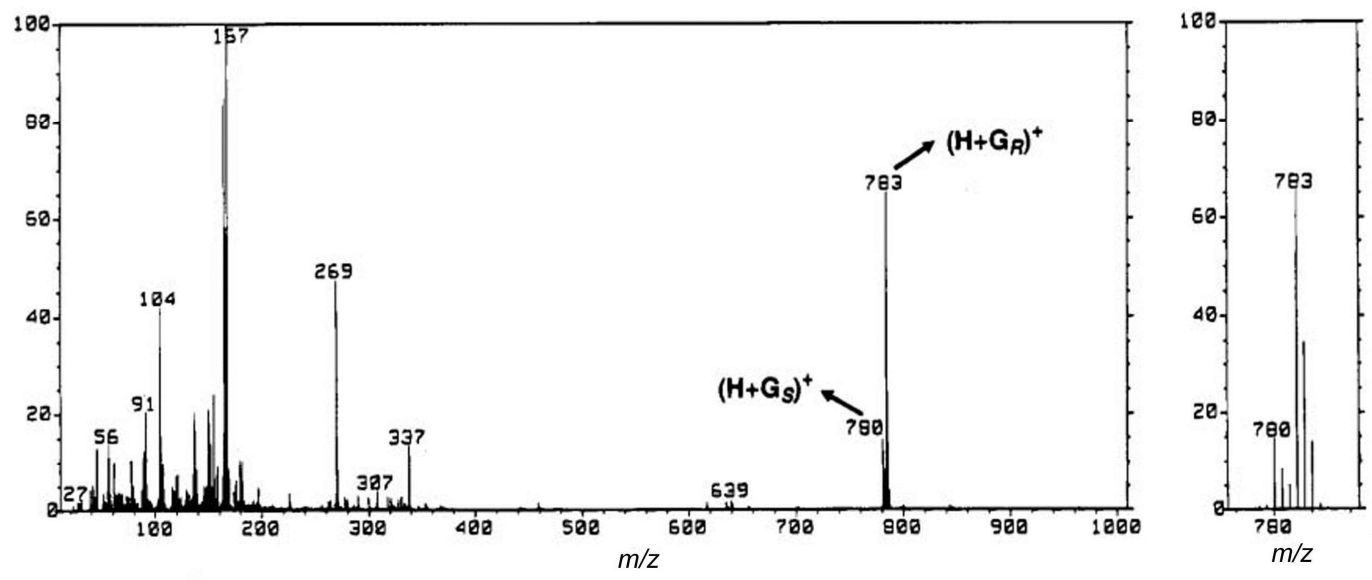

图 37 蛋氨酸甲酯与 66 形成的配合物的 FAB-MS 图

Figure 37 A FAB mass spectrum for the complexation between $\mathbf{6 6}$ and methionine methyl ester<smiles>[R]C(C)Nc1nc(N)nc(N(CCC)CCCC)n1</smiles><smiles>Cc1ccccc1[Se-][Se-]</smiles><smiles>CCCCN(CCC)c1nc(C)nc(N[C](C)c2ccccc2)n1</smiles><smiles>CCCc1ccccc1</smiles>

a: $R, R$

b: $S, S$<smiles>C[C+](N)c1ccccc1</smiles>

$69 \mathrm{a} \sim 69 \mathrm{~b}$

$70 \mathrm{a} \sim 70 \mathrm{~b}$

$$
\text { 71a 71b }
$$<smiles>CC(C)c1ccc(Nc2nc(N)nc(N[C@H](C)c3ccccc3)n2)cc1</smiles><smiles>C[C@H](Nc1nc(N)nc(Nc2ccc(C(C)(C)C)cc2)n1)c1cccc2ccccc12</smiles><smiles>[113In]</smiles><smiles>CC(C)(Nc1nc(N)nc(N)n1)c1ccccc1</smiles>

a: $R, R$

b: $S, S$<smiles>C[C@@H](N)c1ccccc1</smiles>

$74 a \sim 74 b$<smiles>CC(C)(Nc1nc(N)nc(N)n1)c1ccccc1</smiles>

a: $R, R$

b: $S, S$<smiles>C[C@@H](N)c1cccc2ccccc12</smiles>

$75 a \sim 75 b$

图 38 化合物 $67 \sim 75$ 的结构

Figure 38 Structures of compounds $67 \sim 75$
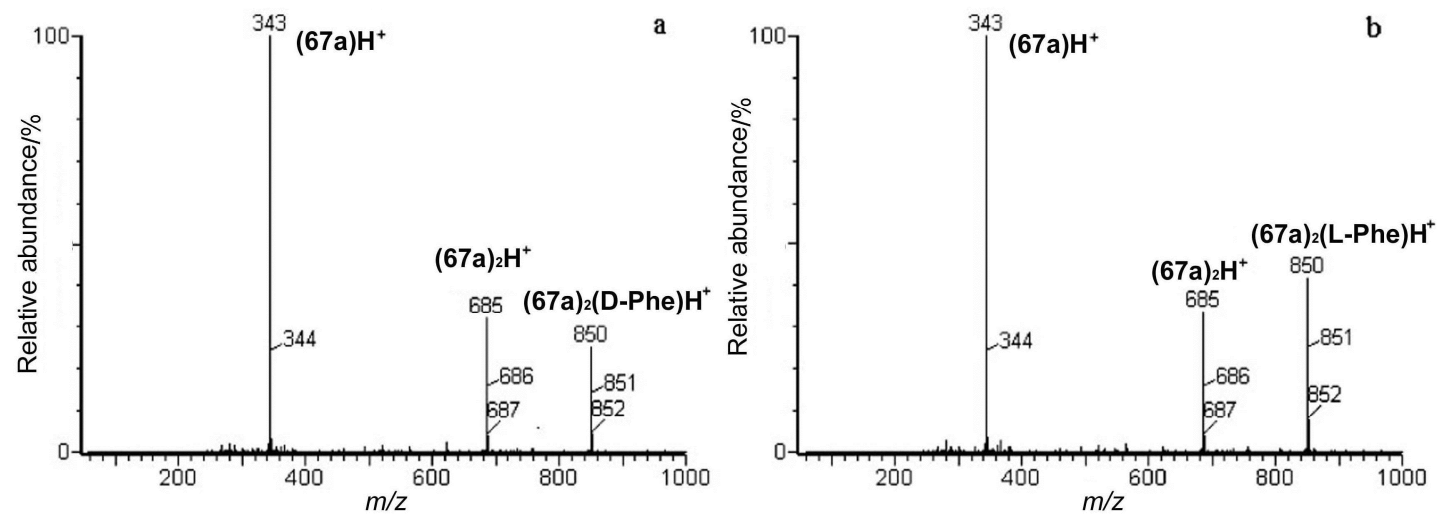

图 39 苯丙氨酸与 $67 \mathrm{a}$ 的两种配合物的 ESI 谱

Figure 39 ESI spectra of two complexes of Phe and 67a

2010 年, $\mathrm{Zu}$ 等 ${ }^{[54]}$ 设计并合成了一系列含羟基脯氨 酸单元的分子钳化合物 77 80(图 41), 通过 ESI-MS 考
察其对一系列二硝基苯(DNB)氨基酸衍生物 $\mathbf{G 5 3} \sim \mathbf{G 6 4}$

(图 42)的手性识别能力, 并对 ESI-MS 的条件进行了优 


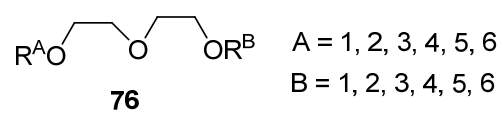

$\mathrm{R}^{1}=\sqrt{\mathrm{R}^{2}=}$<smiles>[R]=C=[R]=CCC[C@H](C)CCC=C(C)C</smiles>

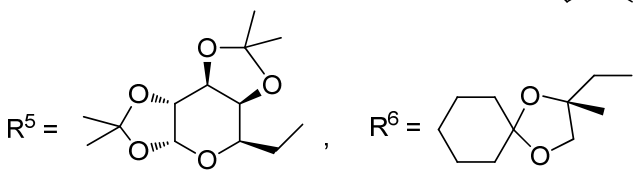

图 40 化合物 76 的结构

Figure 40 Structure of compound 76

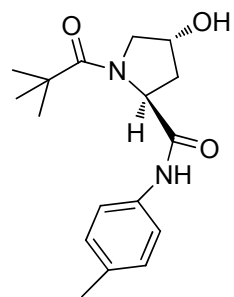

$(2 S, 4 R)-77$

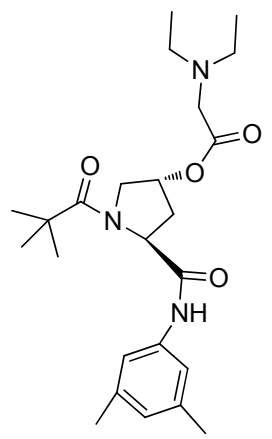

$(2 S, 4 R)-79$

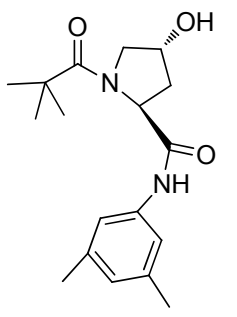

$(2 S, 4 R)-78$

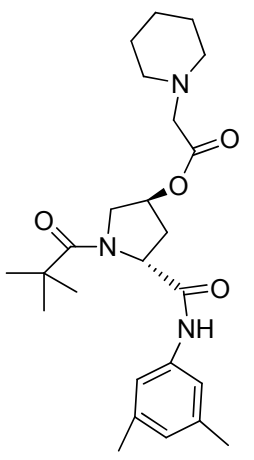

$(2 R, 4 S)-80$

图 41 化合物 $78 \sim 80$ 的结构

Figure 41 Structures of compounds $\mathbf{7 8} \sim \mathbf{8 0}$

化. 实验结果表明, 分子钳 77 $\sim 78$ 上的羟基不利于手性 识别, 分子钳 77 80 上的叔丁基位于反式的 4-羟基泛 氨酸远离苯环的另一端, 不仅有利于电离, 而且提高了 其手性识别的能力. 质谱最佳条件为, 溶剂温度 $150{ }^{\circ} \mathrm{C}$, 氯化铵浓度为 $0.5 \sim 1.0 \mathrm{mmol} / \mathrm{L}$. 同时, 将分子 钳化合物 77 80 制备成手性柱, 通过 HPLC 实验考察其 对一系列二硝基苯(DNB)氨基酸衍生物的分离效果, 然 后与 ESI-MS 结果进行对比, 发现 ESI-MS 不仅速度快, 精确度高, 而且可作为手性选择剂的篮选方法.

2013 年, 左爱平等 ${ }^{[55}$ 利用电喷雾质谱法考察了两 种联萗酫 81 和 82(图 43) 对氨基酸以及氨基醇的手性识 别能力. 主体分子 81 和 82 上的醛基可与氨基酸或氨基 醇上的氨基生成亚胺化合物, 由于反应受到反应速率及 产物稳定性的影响, 手性客体分子与主体分子 81 和 82<smiles>[R]C(=O)C(CC(C)C)NC(=O)c1cc([N+](=O)[O-])cc([N+](=O)[O-])c1</smiles><smiles>[R]C(=O)C(NC(=O)c1cc([N+](=O)[O-])cc([N+](=O)[O-])c1)C(C)C</smiles>

$$
\begin{array}{ll}
\text { G53 } & \mathrm{R}^{1}=\mathrm{NHBu} \\
\text { G54 } & \mathrm{R}^{1}=\mathrm{NEt}_{2} \\
\text { G55 } & \mathrm{R}^{1}=\mathrm{OH}
\end{array}
$$

G56 $\mathrm{R}^{1}=\mathrm{NHBu}$

G57 $\mathrm{R}^{1}=\mathrm{NEt}_{2}$

G58 $\mathrm{R}^{1}=\mathrm{OH}$<smiles>[R]C(=O)C(NC(=O)c1cc([N+](=O)[O-])cc([N+](=O)[O-])c1)c1ccccc1</smiles><smiles>[R]C(=O)C(C)NC(=O)c1cc([N+](=O)[O-])cc([N+](=O)[O-])c1</smiles>

$\begin{array}{ll}\mathbf{G 5 9} & \mathrm{R}^{1}=\mathrm{NHBu} \\ \mathbf{G 6 0} & \mathrm{R}^{1}=\mathrm{NEt}_{2} \\ \mathbf{G 6 1} & \mathrm{R}^{1}=\mathrm{OH}\end{array}$

G62 $\mathrm{R}^{1}=\mathrm{NHBu}$

G63 $R^{1}=\mathrm{NEt}_{2}$

G64 $R^{1}=\mathrm{OH}$

图 42 客体 G53 G64 的结构

Figure 42 Structures of guests $\mathbf{G 5 3} \sim \mathbf{G 6 4}$

的结合当量数不同, 在质谱上两种复合物的离子丰度具 有一定差异. 在 DMSO 中, 主体分子 81 和 82 与单个氨 基的氨基酸(如苯丙氨酸)的质谱显示为, 不匹配的识别 产物离子峰高于匹配识别产物的离子峰, 而对于结构中 含有多个氨基或羧基的氨基酸(如谷氨酸), 则恰恰相反. 加入一定量的氨水并在三乙胺的催化条件下, 氨基酸发 生差向异构化, 质谱峰中匹配识别产物离子峰变得更 高. 电喷雾质谱法对氨基酸以及氨基醇表现出的识别能 力具有普适性, 并认为该质谱主体分子标记法可用于未 知氨基酸及氨基醇的绝对构型的确定.

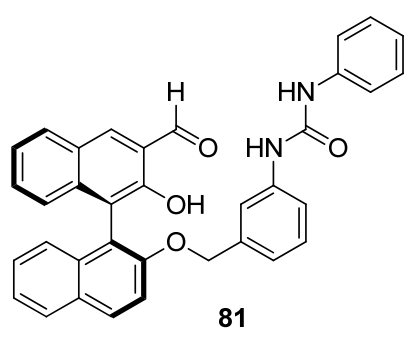

图 43 化合物 $81 \sim 82$ 的结构
2014 年, Fayzullin 等 ${ }^{[56,57]}$ 设计合成了一类套锁型冠 醚 83 87(图 44), 并通过 ESI-MS 同位素标记客体分子 考察其对氨基酸酯的手性选择性. 质谱结果表明, 钳型 受体 84 86 对氨基酸酯表现出较好的手性选择性, 主 体 84 $\sim 86$ 与 $S$ 型氨基酸酯的结合能力强于 $R$ 型. 当冠 醚环变大或在冠醚环上引入芳香基(如钳形受体 86 87), 主体化合物对氨基酸酯结合能力增大,而其手性选 择性却减弱. 主体 84 的手性选择性最好, 相比于 85 , 苯 环上甲氧基的邻位取代的分子主体的手性选择性优于 


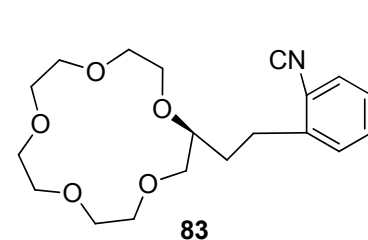<smiles>COc1ccccc1OC[C@H]1COCCOCCOCCOCCO1</smiles>

84

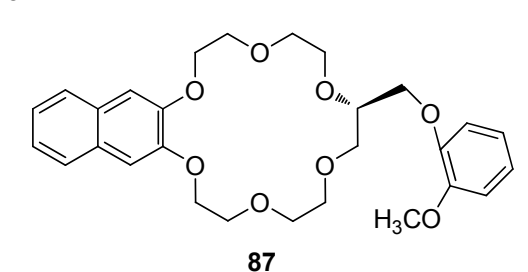

图 44 化合物 $83 \sim 87$ 的结构

Figure 44 Structures of compounds $83 \sim 87$

对位取代的分子主体, 主体 84 和 85 对 $\mathrm{AlaOMe} \cdot \mathrm{HCl}$ 的 $I_{R} / I_{S}$ 分别为 0.61 和 0.99 .

\section{4 基于其他方法的手性选择性研究}

近年来, 随着计算机水平的不断发展, 开始有人利 用分子模拟技术从原子水平解释人工受体模型与被识 别对象之间的手性识别机理 ${ }^{[58 \sim 60]}$. 分子模拟可以直观 给出分子构象, 建立分子识别模型, 通过比较形成配合 物前后, 手性选择剂的变化以及手性选择剂与对映异构 体之间相互作用能的大小, 从而对其产生手性选择性识 别的机理进行解释. 因此, 分子模拟技术作为手性识别 的一个补充, 有利于人们对手性识别的机理进行研究, 从而更好地设计合成出具有良好手性识别能力的人工 受体模型.

2000 年, 杨祖幸等 ${ }^{[61]}$ 设计并合成了系列二聚炔雌 醇分子钳化合物, 并通过紫外-可见光谱滴定考察其对 苯丙氨酸甲酯的手性选择性. 实验研究表明, 该系列分 子钳化合物 88 90 (图 45) 对 $D$-苯丙氨酸甲酯具有更好 的选择性, 其中其 $K_{D} / K_{L}$ 最高达 3.18. 2008 年, 杨祖幸 ${ }^{[62]}$ 等就先前报道的结果, 通过分子力学和分子动力学模拟 解释炔雌醇构筑的分子钳对苯丙氨酸甲酯具有手性识 别能力的作用机理进行解释. 客体分子进入主体分子裂 隙内, 客体的苯环与主体分子钳的芳环产生 $\pi-\pi$ 重叠作 用. 主体分子钳与 $D$-苯丙氨酸甲酯形成的配合物的稳 定常数大于与 $L$-苯丙氨酸甲酯形成的配合物的稳定常 数, 这使得该分子钳化合物对 $D$-苯丙氨酸甲酯的结合 强于 $L$-苯丙氨酸甲酯. 主体分子钳与苯丙氨酸甲酯形成 配合物的相互作用能中, 范德华作用力的比例远大于静 电作用力. 计算机分子模拟技术所得到的结果与紫外可见光谱滴定实验的结果相一致.

Langmuir-Blodgget (LB) 膜技术是一种构建有机有 序超薄分子膜的技术. 与液相不相容的表面活性物质会 在液相表面形成单分子层, 通过增加压力, 使分子紧密

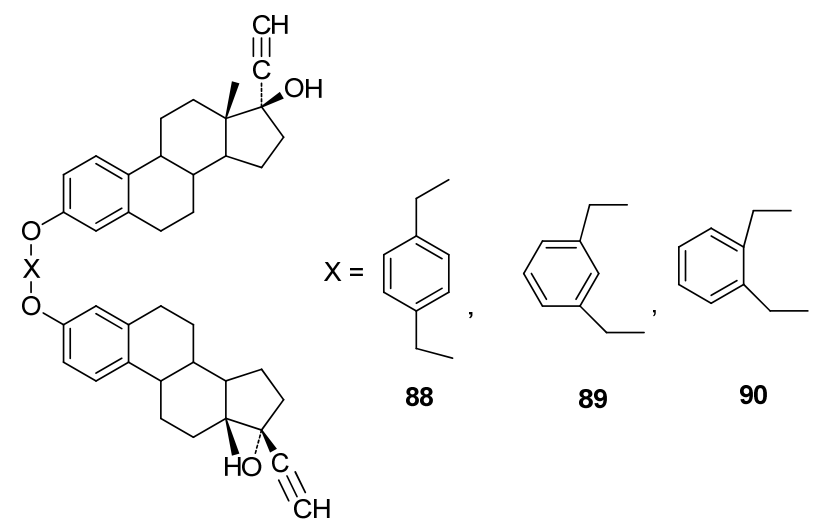

图 45 化合物 $88 \sim 90$ 的结构

Figure 45 Structures of compounds $88 \sim 90$

排列，形成类似固体的单分子层. 这时，将适当的固体 基片浸入或移出液相时, 可把单分子层从液相转移到固 体表面, 即形成 LB 膜. 部分分子钳化合物由于同时具 有疏水基团与亲水基团，可将其与客体分子置于液相中 制成 LB 膜，再与其他技术(如 CD 谱)联用，以实现其手 性识别性能的研究.

2004 年, Liu 等 ${ }^{[63]}$ 设计并合成了三种以杯芳烃为隔 离基，以双环胍盐作为手性臂的分子钳化合物 91 93 (图 46), 并将主体分子钳化合物与苯丙氨酸两性分子制 成 LB 膜，再配合 CD 谱对制成的 LB 膜进行检测，从而 考察主体分子钳化合物对苯丙氨酸的手性识别能力. $\mathrm{CD}$ 谱显示, 分子钳 $91 \sim 93$ 对 $L$-苯丙氨酸的选择性优于 $D$-苯丙氨酸, 且分子钳 91 和 93 对 $L$-苯丙氨酸的选择性 均优于分子钳 92 对 $L$-苯丙氨酸的选择性. 该实验结果 与之前利用液-液竞争萃取实验的结果相一致. 手性识 别过程中, 91 具有钳形结构, 两手臂的双环胍盐的协同 作用形成一个手性环境，由于甲硅烷基醚的疏水性导致 主体分子钳 91 两手臂之间的空隙穴打开，氨基酸分子 可以直接进入空穴内与双环胍盐发生作用，图 47 为分 子钳化合物 91 在空气一水界面的分子结构. 


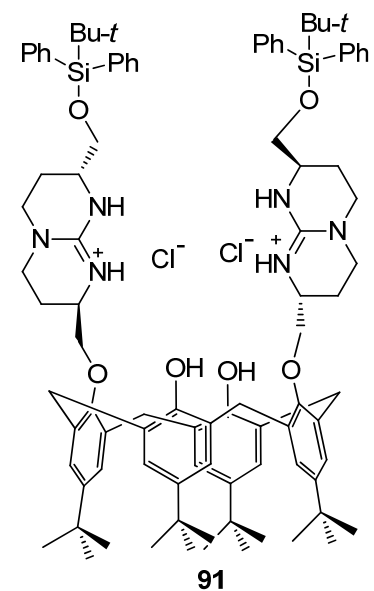

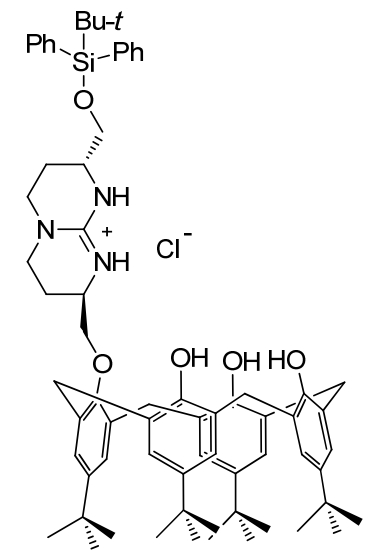

92

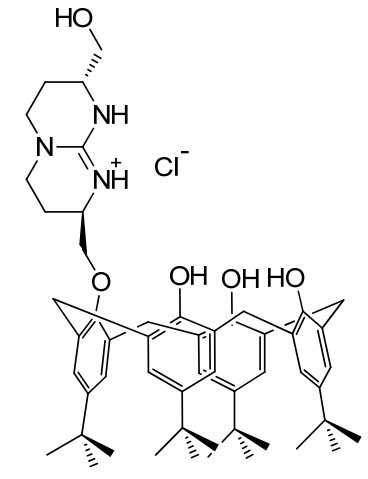

93

图 46 化合物 91 93 的结构

Figure 46 Structures of compounds 91 93

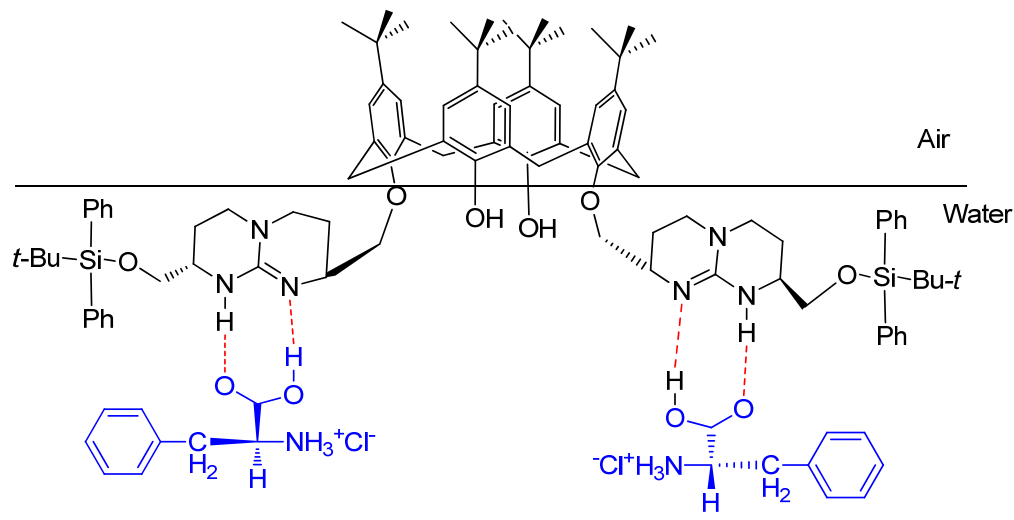

图 47 分子钳化合物 91 在空气一水界面的分子结构

Figure 47 Molecular structures of compound 91 at the air-water interface

2011 年, $\mathrm{Tu}$ 等 ${ }^{[64]}$ 报道了一种通过金属凝胶裂解现 象对钳型受体 94(图 48)的手性识别能力进行考察的方 法. 该方法发现, 金属凝胶 94 加入 $R$ 型的联䒺衍生物经 加热冷却后, 凝胶现象依然存在, 而加入 $S$ 型的联䒺衍 生物经加热冷却后, 凝胶现象消失. $\mathrm{Tu}$ 等认为, 这是由 于不同构型联萘衍生物与金属凝胶的分子间作用力不 同导致. 通过 HR-MS 对此进行验证, 质谱结果显示, 金

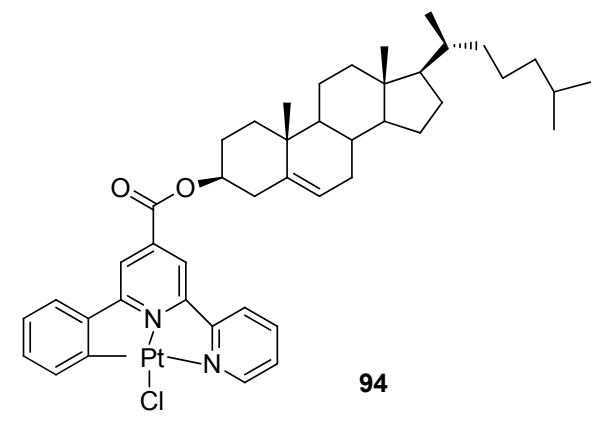

图 48 化合物 94 的结构

Figure 48 The structure of compound 94
属凝胶 94 与 $S$ 型的联萗衍生物形成的配合物离子丰度 高于 $R$ 型的联菜衍生物, 证明金属凝胶 94 与联菱衍生 物对映异构体之间的作用力不同.

\section{2 结语}

随着生命科学的不断发展, 分子识别已成为生物有 机化学和超分子化学的研究热点, 越来越多的用于手性 识别的新型人工受体被合成出来. 分子钳人工受体具有 结构易于修饰的特点, 已成为手性识别研究领域重要的 人工受体之一. 随着超分子化学的不断发展, 越来越多 的分子钳人工受体被合成并应用于手性对映异构体的 识别分离. 文中对近年来分子钳人工受体的合成以及应 用光谱、色谱及质谱等方法对其手性识别性能的研究进 行了总结. 相比于自然界中生物体内精确的分子识别, 分子钳人工受体的合成和性能还显得比较粗楉，合成更 加精准的分子识别主体, 将多种方法联用探究其识别机 理, 进一步了解识别过程中相互作用的推动力与分子结 构之间的关系，将成为手性识别领域的发展方向. 


\section{References}

[1] Berthod, A. Anal. Chem. 2006, 78, 2093.

[2] Qin, H. J.; Yang, X.; He, Y. B.; Qin, Y. G.; Liu, G. J. Acta Chim Sinica 2006, 64, 2271 (in Chinese). (秦海娟，杨洗，何永炳，卿光炎，刘成金，化学学报，2006，64, 2271.)

[3] Qing, G. Y.; Liu, S. Y.; He, Y. B. Prog. Chem. 2008, 20, 1933 (in Chinese).

(卿光炎，刘顺英，何永炳，化学进展, 2008, 20, 1933.)

[4] Berthod, A. Anal. Chem. 2006, 78, 2093.

[5] Rebek, J. Acc. Chem. Res. 1990, 23, 399

[6] Jo, H. H.; Lin, C. Y.; Anslyn, E. V. Acc. Chem. Res. 2014, 47, 2212.

[7] Seifert, H. M.; Jiang, Y. B.; Anslyn, E. V. Chem. Commun. 2014, $50,15330$.

[8] Chen, Y. Z.; Li, H.; Yang, H. J.; Hua, S. M.; Li, H. Q.; Zhao, F. Z.; Chen, N. Y. Org. Mass Spectrom. 1988, 23, 821.

[9] Sawada, M. Mass Spectrom. Rev. 1997, 16, 73

[10] Quinn, T. P.; Atwood, P. D.; Tanski, J. M.; Moore, T. F.; Folmer-Anderson, J. F. J. Org. Chem. 2011, 76, 10020.

[11] Wenzel, T. J.; Amonoo, E. P.; Shariff, S. S.; Aniagyei, S. E. Tetrahedron: Asymmetry 2003, 14, 3099.

[12] Seker, S.; Baris, D.; Arslan, N.; Turgut, Y.; Pirinççioglu, N.; Togrul, M. Tetrahedron: Asymmetry 2014, 25, 411

[13] Nandipati, V.; Akinapelli, K.; Koya, L.; Starnes, S. D. Tetrahedron Lett. 2014, 55, 985.

[14] Yang, K.; Li, S. Z.; Wang, Y. H.; Zhang, W. Z.; Xu, Z. H.; Zhou, X. Y.; Zhu, R. X.; Luo, J.; Wan, Q. RSC Adv. 2014, 4, 6517.

[15] Garcia-Tellado, F.; Albert, J.; Hamilton, A. D. J. Chem. Soc., Chem. Commun. 1991, 1761

[16] Goswami, S.; Ghosh, K.; Dasgupta, S. J. Org. Chem. 2000, 65, 1907.

[17] Liu, S. Y.; Lei, F.; He, Y. B.; Chan, W. H.; Yeung, K. T.; Cheng, Y. K.; Yang, R-H. Org. Lett. 2005, 7, 5825.

[18] Li, S. Y.; Zheng, Q. Y.; Chen, C. F.; Huang, Z. T. Tetrahedron: Asymmetry 2005, 16, 2816.

[19] Qing, G. Y.; He, Y. B.;Wang, F.; Qin, H. J.; Hu, C. G.; Yang, X. Eur. J. Org. Chem. 2007, 11, 1768 .

[20] Qing, G. Y.; Qin, H. J.; He, Y. B.; Hu, C. G.; Wang, F.; Hu, L. Supramol. Chem. 2008, 20, 265.

[21] Feng, Z. Q. M.S. Thesis, Suzhou University, Suzhou, 2012 (in Chinene). (冯志强, 硕士论文, 苏州大学, 苏州, 2012.)

[22] Jiang, J. X. Ph.D. Dissertation, Suzhou University, Suzhou, 2013 (in Chinese). (蒋佳珣, 博士论文, 苏州大学, 苏州, 2013.)

[23] Granda, J. M.; Jurczak, J. Org. Lett. 2013, 15, 4730.

[24] Granda, J. M.; Jurczak, J. Chem. Eur. J. 2014, 20, 12368.

[25] Granda, J. M.; Jurczak, J. Chem. Eur. J. 2015, 21, 16585.

[26] Li, Z. Y.; Zhou, K.; Lai, Y.; Sun, X. Q.; Wang, L. Y. Chin. J. Org. Chem. 2015, 35, 1531 (in Chinese).

(李正义, 周坤, 来源, 孙小强, 王乐勇, 有机化学, 2015, 35, 1531.)

[27] Gao, G.; Lv, C.; Li, Q.; Ai, L.; Zhang, J. Tetrahedron Lett. 2015, 56, 6742 .

[28] González-Mendoza, L.; Escorihuela, J. Altava, B.; Burguete, M. I.; Luis, S. V. Org. Biomol. Chem. 2015, 13, 5450

[29] Feng, H. T.; Zhang, X.; Zheng, Y. S. J. Org. Chem. 2015, 80, 8096.

[30] Wen, K.; Yu, S.; Huang, Z.; Chen, L.; Xiao, M.; Yu, X.; Pu, L. J. Am. Chem. Soc. 2015, 137, 4517.

[31] Fuentes, A. L.; Herrero, A, G.; Rubio, O. H.; Monleon, L. M.; Rubio, L, S.; Alcazar, V.; Sanz, F.; Moran, J. R. Org. Biomol. Chem.
2015, 13, 493

[32] Botha, F.; Budka, J.; Eigner, V.; Hudecek, O.; Vrzal, L.; Cisarova, I.; Lhotak, P. Tetrahedron 2014, 70, 477.

[33] Mackova, M.; Miksatko, J.; Budka, J.; Eigner, V.; Curinova, p.; Lhotak, P. New J. Chem. 2015, 39, 1382.

[34] Lammerhofer, M.; Svec, F.; Frechet, J. M. J.; Lindner, W. TrAC, Trends Anal. Chem. 2000, 19, 676

[35] Wang, W. Y. M.S. Thesis, Shanxi University, Taiyuan, 2009 (in Chinese).

(王文艳, 硕士论文, 山西大学, 太原, 2009.)

[36] Hernfindez, J. V.; Almaraz, M.; Raposo, C.; Martin, M.; Lithgow, A.; Crego, M.; Caballero, C.; Moran, J. R. Tetrahedron Lett. 1998, 39,7401 .

[37] Martin, M.; Raposo, C.; Oliva, A. L.; Simon, L.; Caballlero, C.; Moran, J. Chem. Lett. 2000, 0, 718.

[38] Gazic, I.; Kontrec, D.; Lesac, A.; Vinkovic, V. Tetrahedron: Asymmetry 2005, 16, 1175 .

[39] Choi, H. J.; Ha, H. J.; Shin, M. S.; Jin, J. S.; Hyun, M. H. J. Liq. Chromatogr. Related Technol. 2009, 32, 1879

[40] Yu, H.; Yin, C.; Jia, C.; Jin, Y.; Ke, Y.; Liang, X. Chirality 2012, 24, 391.

[41] Wu, J.; Su, P.; Guo, D.; Huang, J.; Yang, Y. New J. Chem. 2014, 38, 3630.

[42] Zhou, J.; Wang, Y.; Liu, Y.; Tang, J.; Tang, W. Anal. Chim. Acta $\mathbf{2 0 1 5}, 868,73$

[43] Wernisch, S.; Pell, R.; Lindner, W. J. Sep. Sci. 2012, 35(13), 155.

[44] Hyum, M. H.; Jin, J, S.; Lee, W. J. Chromatogr. A 1998, 822, 391.

[45] Lu, Z.; Wu, P.; Zi, M.; Yang, C.; Kong, J.; Yuan, L. Chin. J. Org. Chem. 2015, 35, 217.

[46] Wang, D.; Zhao, J.; Wu, H.; Wu, H.; Cai, J.; Ke, Y.; Liang, X. J. Sep. Sci. 2015, 38, 205.

[47] Schug, K. A.; Lindner, W. J. Sep. Sci. 2005, 28, 1932.

[48] Ranc, V.; Havlicek, V.; Bednar, P.; Lemr, K. Eur. J. Mass Spectrom. 2008, 14,411

[49] Chen, Y.; Li, H.; Yang, H.; Hua, S.; Li, H.; Zhao, F.; Chen, N. Org. Mass Spectrom. 1988, 23, 821.

[50] Sawada, M.; Okumura, Y.; Shizuma, M.; Takai, Y.; Hidaka, Y.; Yamada, H.; Tanaka, T.; Kaneda, T.; Hirose, K. J. Am. Chem. Soc. 1993, 115,7381 .

[51] Sawada, M.; Takai, Y.; Yamada, H.; Hirayama, S.; Kaneda, T.; Tanaka, T.; Kamada, K.; Mizooku, T.; Takeuchi, S.; Ueno, K.; Hirose, K.; Tobe, Y.; Naemura, K. J. Am. Chem. Soc. 1995, 117, 7726.

[52] Liu, Q.; Zhang, S.; Wu, B.; Guo, J.; Xie, J.; Gu, M.; Zhao, Y.; Yun, L.; Liu, K. Anal. Chem. 2005, 77, 5302.

[53] Shizuma, M.; Adachi, H.; Ono, D.; Sato, H.; Nakamura, M. Chirality 2009, 21, 324.

[54] Zu, C. L.; Woolfolk, J, A.; Koscho, M. E. Anal. Chim. Acta 2010 661,60 .

[55] Zuo, A. P. M.S. Thesis, Central China Normal University, Wuhan, 2013 (in Chinese) (左爱平，硕士论文，华中师范大学，武汉，2013.)

[56] Fayzullin, R. R.; Bredikhina, Z. A.; Sharafutdinova, D. R.; Bazanova, O. B.; Bredikhin, A. A. Russ. J. Org. Chem. 2014, 50, 611.

[57] Bredikhina, Z. A.; Sharafutdinova, D. R.; Bazanova, O. B.; Babaev, V. M.; Fayzullin, R. R.; Rizvanov, I. K.; Bredikhin, A. A. J. Incl. Phenom. Macrocycl. Chem. 2014, 80, 417.

[58] Alexander, J. M.; Clark, J. L.; Brett, T. J.; Stezowski, J. J. Proc. Natl. Acad. Sci. U. S. A. 2002, 99, 5115.

[59] Nunez-Aguero, C. J.; Escobar-Llanos, C. M.; Diaz, D.; Jaime, C.; Garduno-Juarez, R. Tetrahedron 2006, 62, 4162.

[60] Zhao, C.; Cann, N. M. J. Chromatogr. A 2007, 1149, 197.

[61] Yang, Z. Y.; Liu, L.; Cheng, Y.; Wang, Y. L.; Chen, S. H.; Gan, Y.; 
Lv, D. J. Sichuan Univ. (Nat. Sci. Ed.) 2000, 37, 477 (in Chinese). (杨祖幸, 刘丽, 程勇, 王玉良, 陈淑华, 甘亚, 吕丁, 四川大学 学报 (自然科学版), 2000, 37, 477.)

[62] Yang, Z. Y.; Zhang, W. H. Chem. Res. Appl. 2008, 20, 386 (in Chinese)
(杨祖幸, 张文华, 化学研究与应用, 2008, 20,386.)

[63] Liu, F.; Lu, G. Y.; He, W. J.; Liu, M. H.; Zhu, L. G. Thin Solid Films 2004, 468, 244.

[64] Tu, T.; Fang, W.; Bao, X.; Li, X.; Dotz, K. H. Angew. Chem., Int. Ed. 2011, 50, 6601.

(Cheng, F.) 